\title{
Aperture-free star formation rate of SDSS star-forming galaxies ${ }^{\star}$
}

\author{
S. Duarte Puertas ${ }^{1}$, J. M. Vilchez ${ }^{1}$, J. Iglesias-Páramo ${ }^{1,2}$, C. Kehrig ${ }^{1}$, E. Pérez-Montero ${ }^{1}$, and F. F. Rosales-Ortega ${ }^{3}$ \\ ${ }^{1}$ Instituto de Astrofísica de Andalucía - CSIC, Glorieta de la Astronomía s.n., 18008 Granada, Spain \\ e-mail: salvini@iaa.es \\ 2 Estación Experimental de Zonas Áridas - CSIC, Ctra. de Sacramento s.n., La Cañada, 04120 Almería, Spain \\ ${ }^{3}$ Instituto Nacional de Astrofísica, Óptica y Electrónica, Luis E. Erro 1, 72840 Tonantzintla, Puebla, Mexico
}

Received 2 June 2016 / Accepted 15 November 2016

\begin{abstract}
Large area surveys with a high number of galaxies observed have undoubtedly marked a milestone in the understanding of several properties of galaxies, such as star-formation history, morphology, and metallicity. However, in many cases, these surveys provide fluxes from fixed small apertures (e.g. fibre), which cover a scant fraction of the galaxy, compelling us to use aperture corrections to study the global properties of galaxies. In this work, we derive the current total star formation rate (SFR) of Sloan Digital Sky Survey (SDSS) star-forming galaxies, using an empirically based aperture correction of the measured $\mathrm{H} \alpha$ flux for the first time, thus minimising the uncertainties associated with reduced apertures. All the $\mathrm{H} \alpha$ fluxes have been extinction-corrected using the $\mathrm{H} \alpha / \mathrm{H} \beta$ ratio free from aperture effects. The total SFR for $\sim 210000$ SDSS star-forming galaxies has been derived applying pure empirical H $\alpha$ and $\mathrm{H} \alpha / \mathrm{H} \beta$ aperture corrections based on the Calar Alto Legacy Integral Field Area (CALIFA) survey. We find that, on average, the aperture-corrected SFR is $\sim 0.65$ dex higher than the SDSS fibre-based SFR. The relation between the SFR and stellar mass for SDSS star-forming galaxies $\left(S F R-M_{\star}\right)$ has been obtained, together with its dependence on extinction and $\mathrm{H} \alpha$ equivalent width. We compare our results with those obtained in previous works and examine the behaviour of the derived SFR in six redshift bins, over the redshift range $0.005 \leq z \leq 0.22$. The $S F R-M_{\star}$ sequence derived here is in agreement with selected observational studies based on integral field spectroscopy of individual galaxies as well as with the predictions of recent theoretical models of disc galaxies.
\end{abstract}

Key words. galaxies: general - galaxies: star formation - galaxies: formation - galaxies: evolution

\section{Introduction}

In the last two decades we have witnessed the revolutionary appearance of large area surveys with a huge number of galaxies observed (e.g. Sloan Digital Sky Survey (SDSS), York et al. 2000; 2dFGRS, Colless et al. 2001; VVDS, Le Fèvre et al. 2005; GAMA, Driver et al. 2011). These surveys have been very useful for performing a complete study of the non-uniform distribution of galaxies in the Universe (large-scale structure) and to understand galaxy formation and evolution. They also give us information about important properties of galaxies, such as morphology, stellar mass, star formation rate, metallicity, and dependence on the environment. These surveys used singlefibre spectroscopy with small apertures (e.g. 2" diameter for 2dFGRS and GAMA, and 3" diameter for SDSS) and therefore cover a limited region of the galaxy, thus providing partial information on the extensive properties of galaxies. For a more detailed analysis of the integrated properties of each galaxy, it is necessary to make use of integral field spectrographs (IFS, e.g. Kehrig et al. 2012, 2016). IFS surveys, such as SAURON (Bacon et al. 2001), PINGS (Rosales-Ortega et al. 2010), MASSIV (Contini et al. 2012), CALIFA (Sánchez et al. 2012; García-Benito et al. 2015), and MANGA (Bundy et al. 2015), are based on arrays of fibres and allow us to obtain information from the whole galaxy. However, the integration times

\footnotetext{
* A table of the aperture-corrected fluxes and SFR for $\sim 210000$ SDSS star-forming galaxies and related relevant data is only available at the CDS via anonymous ftp to cdsarc.u-strasbg. fr $(130.79 .128 .5)$ or via http://cdsarc.u-strasbg.fr/viz-bin/qcat?J/A+A/599/A71
}

necessary to observe each galaxy are long, hindering the acquisition of a large number of galaxies in comparison with that obtained from single-fibre surveys.

It is clear that to gain a better insight into the global properties of galaxies we need tools that allow us to link both singlefibre and IFS surveys. In this work we make use of one of these tools for the purpose of studying the total current star formation rate (hereafter SFR) of star-forming galaxies, using their total $\mathrm{H} \alpha$ fluxes emitted by the gas ionised by young massive stars (e.g. Kennicutt 1998; Kennicutt et al. 2009). The SFR presents a well-known characteristic relation with the stellar mass (e.g. Brinchmann et al. 2004b). In the SFR-stellar mass plane $\left(S F R-M_{\star}\right)$, active star-forming galaxies define a distinct sequence called main sequence (Noeske et al. 2007). In addition, less active star-forming galaxies (e.g. quenched and ageing starforming galaxies) appear in this plane located at higher masses and lower SFR values (e.g. Renzini \& Peng 2015; Casado et al. 2015; Leslie et al. 2016), as well as a family of outliers to this main sequence (MS) that are generally interpreted as starbursts driven by merging (e.g. Rodighiero et al. 2011). The MS of star formation has been parametrized by the following equation:

$\log (S F R)=\alpha \log \left(M_{\star}\right)+\beta$.

From a theoretical point of view, recent studies have obtained values for $\alpha$ (MS slope) near to unity (e.g. Dutton et al. 2010; Sparre et al. 2015; Tissera et al. 2016). Dutton et al. (2010) used a semi-analytic model of disc galaxies parametrizing several properties: for example, SFRs and metallicities were computed in a spatially resolved way as a function of galactic radius. For galaxies with stellar masses between $10^{9} M_{\odot}$ and $10^{11} M_{\odot}$ the 
slope found in Dutton et al. (2010) is $\alpha=0.96$. In contrast, Sparre et al. (2015) used Illustris (state-of-the-art cosmological hydrodynamical simulation of galaxy formation; Nelson et al. 2015) to reproduce the observed star formation MS, finding a similar slope to Dutton et al. (2010) at low redshift. From observational studies quoting $\mathrm{H} \alpha$ based SFR determinations, the MS slope varies preferentially between $\sim 0.6$ and $\sim 1$, and $\beta$ between $\sim-9$ and $\sim-3$, depending on the precise methodology and data used (see e.g. Rodighiero et al. 2011; Speagle et al. 2014, and references therein). An important factor behind this spread in $\alpha$ and $\beta$ values can be related to the aperture corrections applied to the $\mathrm{H} \alpha$ measurements.

In order to evaluate the SFR of a galaxy in single-fibre surveys, an aperture correction needs to be applied to account for the entire galaxy. This correction becomes essential in order to analyse the SFR dependence with redshift $(z)$, especially for samples of star-forming galaxies at low $z$. Many such studies have dealt with the SFR of star-forming galaxies in the local Universe (e.g. Brinchmann et al. 2004b; Iglesias-Páramo et al. 2006; Salim et al. 2007; Kennicutt et al. 2008; Peng et al. 2010) and others extended to medium and large redshift (e.g. Madau et al. 1996; Elbaz et al. 2007; Peng et al. 2010; Whitaker et al. 2012; Drake et al. 2013, 2015). At lower redshift the effects produced by a fixed aperture size will be clearly more significant than for high redshifts. It is important to note that for galaxies about the size of the Milky Way, the 3-arcsec-diameter SDSS fibre never encompasses the complete galactic disc. For this reason, it is always necessary to use aperture corrections to derive the total SFR when using the SDSS $\mathrm{H} \alpha$ fluxes. An extra drawback produced by this limitation of the SDSS fibre is particularly relevant for "late-type" spiral galaxies (i.e. disc-dominated galaxies with Hubble type from $\mathrm{Sb}$ to Sdm, Dahlem 1997) since they present higher star formation in the outer parts of their discs. Therefore, the $\mathrm{H} \alpha$ flux measured by SDSS for these galaxies would lead to an underestimation of the total SFR.

Several studies have already emphasised the importance of quantifying the effect of aperture in the observational data (e.g. Kewley et al. 2005; Kennicutt et al. 2008; Mast et al. 2014) and also when comparing with the theoretical model predictions (Guidi et al. 2016). To our knowledge, a solid empirical aperture correction has not yet been implemented in a systematic way for the analysis of large samples of galaxies. Up to now, most studies quantifying the total SFR of galaxies from single-fibre surveys apply aperture corrections using model-based methods (e.g. Brinchmann et al. 2004b; Salim et al. 2007). Other works apply geometrical considerations in order to compensate for the unobserved $\mathrm{H} \alpha$ emission of the galaxy, scaling it according to its broad-band photometric map, or by using analytical recipes (e.g. Hopkins et al. 2003, 2013). For SDSS star-forming galaxies, Brinchmann et al. (2004b) originally corrected SDSS fibre SFRs from aperture effects using the resolved colour information available for each galaxy. In the Max-Planck-Institut für Astrophysik and Johns Hopkins University (MPA-JHU) database $^{1}$ (Kauffmann et al. 2003b; Brinchmann et al. 2004b; Tremonti et al. 2004; Salim et al. 2007) the Brinchmann et al. (2004b) methodology, improved following Salim et al. (2007), was used to derive SFRs. It is important to note that MPA-JHU galactic SFRs always include the nuclear emission. In addition, the median profiles of the growth curve corresponding to the $\mathrm{H} \alpha / \mathrm{H} \beta$ aperture correction decreases when galaxy radius increases (Iglesias-Páramo et al. 2013). Thus, galaxies for which

Available at http://www .mpa-garching.mpg.de/SDSS/ only the central zones are observed present overestimated extinction (Iglesias-Páramo et al. 2016, hereafter IP16): on average, some bias is expected in MPA-JHU SFRs since galaxies' extinction gradients were not accounted for (Richards et al. 2016).

A rigorous methodology to derive the total SFR of a galaxy should make use of its entire $\mathrm{H} \alpha$ flux and $\mathrm{H} \alpha / \mathrm{H} \beta$ ratio. For single-fibre surveys, the total $\mathrm{H} \alpha$ flux of a galaxy can be obtained using an empirical aperture correction for $\mathrm{H} \alpha$ derived from IFS of nearby galaxies. According to Iglesias-Páramo et al. (2013, 2016), the CALIFA project allows an accurate aperture correction for $\mathrm{H} \alpha$ to be derived empirically. IP16 provide the growth curve of $\mathrm{H} \alpha$ flux as a function of $R_{50}$, the Petrosian radius containing $50 \%$ of the total galaxy flux in the $r$-band, on the basis of a representative sample of 165 star-forming galaxies from the CALIFA survey (Sánchez et al. 2012; Husemann et al. 2013; Walcher et al. 2014). In this work we make use of the empirical aperture correction from IP16 in order to obtain the total $\mathrm{H} \alpha$ flux for a sample of 210000 star-forming galaxies from the SDSS. From this total $\mathrm{H} \alpha$ flux we derive aperture-corrected values of SFR in each galaxy, which will allow us to study the global relation between the SFR, stellar mass, and redshift.

To our knowledge this work represents the first attempt to analyse the behaviour of the present-day SFR for a large sample including all SDSS star-forming galaxies using the total $\mathrm{H} \alpha$ emission empirically corrected by $\mathrm{H} \alpha$ aperture coverage in a systematic manner. The structure of this paper is organised as follows. In Sect. 2 we describe the data and provide a description of the methodology used to select the star-forming galaxies. We detail the methodology used to derive the entire $\mathrm{H} \alpha$ flux using the empirical aperture correction and the corresponding SFR in Sect. 3. Our main results are presented in Sect. 4 and the associated discussion in Sect. 5. Finally, a summary and the main conclusions of our work are given in Sect. 6.

Throughout the paper, we assume a Friedman-RobertsonWalker cosmology with $\Omega_{\Lambda 0}=0.7, \Omega_{\mathrm{m} 0}=0.3$, and $H_{0}=$ $70 \mathrm{~km} \mathrm{~s}^{-1} \mathrm{Mpc}^{-1}$. We use the Kroupa (2001) universal initial mass function (IMF) ${ }^{2}$.

\section{Data and sample}

\subsection{Sample selection}

Our study is based on the MPA-JHU public catalogue (Kauffmann et al. 2003b; Brinchmann et al. 2004b; Tremonti et al. 2004; Salim et al. 2007), which gives spectroscopic data of the galaxies in the Sloan Digital Sky Survey Data Release 7 (SDSS-DR7) (Abazajian et al. 2009). The SDSS spectroscopic primary sample of galaxies is complete in Petrosian $r$ magnitude in the range $14.5 \leq r \leq 17.7$ (Strauss et al. 2002; Brinchmann et al. 2004a). We added the photometric data from the SDSS-DR12 (York et al. 2000; Alam et al. 2015) to the MPA-JHU catalogue. The MPA-JHU catalogue contains 1477411 objects, of which 933310 are galaxies with spectroscopic properties. In this catalogue, the stellar masses are estimated using spectral energy distribution (SED) fitting to ugriz photometry as described in Kauffmann et al. (2003b). The line fluxes ${ }^{3}$ are corrected for foreground Galactic reddening and extinction using the attenuation curve from O'Donnell (1994)

\footnotetext{
2 It is necessary to multiply the Kroupa (2001) SFR estimation by 1.6 to transform from the Kroupa (2001) IMF to the Salpeter (1955) IMF and by 0.943 to transform from Kroupa (2001) to Chabrier (2003) IMF (Marchesini et al. 2009; Mannucci et al. 2010).

3 MPA-JHU re-normalised its flux outputs to match the photometric fibre magnitude in the $r$-band. See the MPA-JHU website.
} 


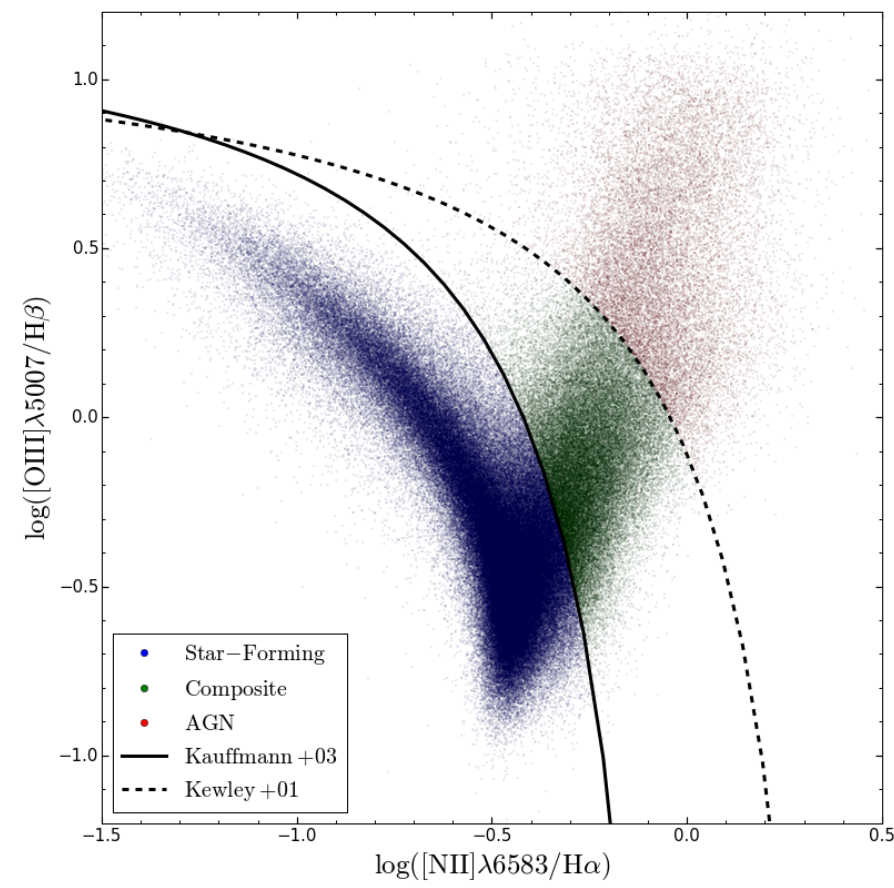

Fig. 1. [OIII] $\lambda 5007 / \mathrm{H} \beta$ versus [NII] $\lambda 6583 / \mathrm{H} \alpha$ diagnostic diagram (BPT) for the SDSS galaxies. Blue, green, and red points represent star-forming galaxies in the present work (209276), composite galaxies (57926), and AGN galaxies (19392), respectively. The dashed line shows the Kewley et al. (2001) demarcation and the continuous line shows the Kauffmann et al. (2003a) curve.

and the extinction from Schlegel et al. (1998). Finally, MPAJHU provides the emission line fluxes measured using Gaussian fittings over the subtracted continuum and the signal-to-noise $(\mathrm{S} / \mathrm{N})$ in the whole spectrum. For those galaxies with multiple entries we selected only those with the largest $\mathrm{S} / \mathrm{N}$ in the whole spectrum, reducing the total number of galaxies to 874701 .

We selected our primary sample according to the following criteria:

i) The galaxy stellar mass $\left[\log \left(M_{\star} / M_{\odot}\right)\right]$ is selected in the range between 8.50 and 11.50 . Galaxies without any estimation of the stellar mass are discarded in order to carry out a proper comparison between the SFR and the stellar mass.

ii) We restricted our sample to the galaxies with small relative error of size measurements of $R_{50}\left(\Delta\left(R_{50}\right) / R_{50} \leq 1 / 3\right)$. In agreement with this consideration, we removed those galaxies with $\Delta\left(R_{50}\right) / R_{50}$ greater than $1 / 3$, which may be detrimental for our study when we use the growth curves from IP16 in order to derive the aperture corrected $\mathrm{H} \alpha$ flux (as will be explained in Sect. 3.1).

iii) We considered galaxies with values for $1.5^{\prime \prime} / R_{50}$ higher than 0.3 (i.e. $R_{50} \leq 5^{\prime \prime}$ ), where $1.5^{\prime \prime}$ is the radius of the SDSS fibre. Iglesias-Páramo et al. (2013) showed that the seeing may affect the Sérsic profile for values below this cut-off limit for the parameter $1.5^{\prime \prime} / R_{50}$, according to the analytical study carried out by Trujillo et al. (2001). The full width at half maximum (FWHM) of the point spread function (PSF) has a median value of $\sim 3.6^{\prime \prime}$ in the CALIFA observations (Husemann et al. 2013; Iglesias-Páramo et al. 2013). iv) We did not consider galaxies whose SDSS spectra were classified as quasi stellar objects (QSO). In the original catalogue we have found 14476 QSO galaxies.

v) Spectroscopic redshift in the range $0.005 \leq z \leq 0.22$. The lower $z$-limit of 0.005 was adopted in order to: a) minimise the effect in the photometric measurements for the larger nearby galaxies; b) to include galaxies with the lowest luminosities (Brinchmann et al. 2004b). It is expected that a sizeable fraction of the low luminosity SDSS galaxies could remain unobserved for the redshift range considered (e.g. Blanton et al. 2005).

Our resulting primary sample contains 655734 galaxies $(74.97 \%$ of the original catalogue).

\subsection{Selection of the star-forming galaxies}

We selected a subset of 209276 star-forming galaxies from our primary sample described in Sect. 2.1 (31.92\% from our primary sample in Sect. 2.1) applying the following criteria:

i) The $\mathrm{S} / \mathrm{N}$ is greater than three for the fluxes in the strong emission lines $\mathrm{H} \alpha, \mathrm{H} \beta$, [OIII], and [NII]. We have defined the $\mathrm{S} / \mathrm{N}$ as the ratio of the flux and the statistical error flux, calculated with the pipeline described in Tremonti et al. (2004) (Kewley \& Ellison 2008). Below this limit in $\mathrm{S} / \mathrm{N}$, an important fraction of galaxies present negative line fluxes (Brinchmann et al. 2004b).

ii) We selected the pure star-forming galaxies according to the Kauffmann definition in the BPT diagnostic diagram (e.g. Baldwin et al. 1981; Veilleux \& Osterbrock 1987; Kewley et al. 2001; Kauffmann et al. 2003a): [OIII] $\lambda 5007 / \mathrm{H} \beta$ versus [NII] $\lambda 6583 / \mathrm{H} \alpha$ (see Fig. 1):

$$
\log ([\mathrm{OIII}] / \mathrm{H} \beta) \leq \frac{0.61}{[\log ([\mathrm{NII}] / \mathrm{H} \alpha)-0.05]}+1.3
$$

$\log ([\mathrm{OIII}] / \mathrm{H} \beta)$ values below this curve imply a contribution to $\mathrm{H} \alpha$ from active galactic nuclei (AGN) less than $1 \%$, (i.e. discarding composite or AGN galaxies; Kauffmann et al. 2003a; Brinchmann et al. 2004b $)^{4}$.

iii) The $\mathrm{H} \alpha$ equivalent width $(E W(\mathrm{H} \alpha))^{5}$ is greater than or equal to three. This condition is assumed to avoid passive galaxies as defined by Cid Fernandes et al. (2011).

In Table 1 we present the median $( \pm 1 \sigma \text { confidence interval })^{6}$ of several parameters split into six redshift bins within the range $0.005 \leq z \leq 0.22$. In Col. 1 the $\Delta z$ is presented, Col. 2 shows the $\log \left(M_{\star} / M_{\odot}\right)$, Cols. 3 and 4 show the $\mathrm{S} / \mathrm{N}$ for the $\mathrm{H} \alpha$ emission line flux and the $\mathrm{S} / \mathrm{N}$ per whole spectrum range, respectively; the $E W(\mathrm{H} \alpha)$ is presented in Col. 5, Col. 6 displays the $R_{50}$, and the number of galaxies per redshift bin is quoted in Col. 7 .

\footnotetext{
4 Galaxies classified as AGN or composite using the SDSS fibre spectra and hosting star formation throughout their discs could be missed in the construction of the final sample.

5 The $E W(\mathrm{H} \alpha)$ is defined as the ratio between the $\mathrm{H} \alpha$ flux and the continuum flux near to $\mathrm{H} \alpha$. For the sake of simplicity in this work we assume $|E W(\mathrm{H} \alpha)|$.

6 The definition of $\pm 1 \sigma$ used in this work is: $+1 \sigma=$ percentile $84-$ median; $-1 \sigma=$ median - percentile 16 .
} 
Table 1. Values of relevant parameters corresponding to the median $( \pm 1 \sigma$ confidence interval) of the distribution for the star-forming galaxies in six redshift bins up to $z=0.22$ in the total sample.

\begin{tabular}{c|cccccc}
\hline \hline$(1)$ & $(2)$ & $(3)$ & $(4)$ & $(5)$ & $(6)$ & $(7)$ \\
$\Delta z$ & $\log \left(M_{\star} / M_{\odot}\right)$ & $S / N F(\mathrm{H} \alpha)$ & $S / N$ (whole spec.) & $E W(\mathrm{H} \alpha)[\AA]$ & $R_{50}\left[^{\prime \prime}\right]$ & \# galaxies \\
\hline $0.005-0.05$ & $9.34_{-0.46}^{+0.56}$ & $67.16_{-29.24}^{+34.51}$ & $12.42_{-5.20}^{+8.29}$ & $24.31_{-11.56}^{+23.47}$ & $3.11_{-1.14}^{+1.14}$ & 41883 \\
$0.05-0.08$ & $9.93_{-0.40}^{+0.43}$ & $63.57_{-23.68}^{+30.35}$ & $11.87_{-4.18}^{+5.94}$ & $23.17_{-10.42}^{+20.13}$ & $2.63_{-0.86}^{+1.01}$ & 62616 \\
$0.08-0.11$ & $10.25_{-0.36}^{+0.34}$ & $61.08_{-22.86}^{+29.58}$ & $11.52_{-3.54}^{+4.81}$ & $22.75_{-10.07}^{+19.62}$ & $2.31_{-0.67}^{+0.77}$ & 48178 \\
$0.11-0.14$ & $10.48_{-0.36}^{+0.30}$ & $55.29_{-21.32}^{+28.74}$ & $11.14_{-3.25}^{+4.05}$ & $23.52_{-10.43}^{+20.75}$ & $2.10_{-0.58}^{+0.64}$ & 31084 \\
$0.14-0.18$ & $10.62_{-0.44}^{+0.28}$ & $55.04_{-22.13}^{+29.99}$ & $10.45_{-3.28}^{+3.64}$ & $25.90_{-11.96}^{+26.52}$ & $1.92_{-0.59}^{+0.60}$ & 17465 \\
$0.18-0.22$ & $10.70_{-0.50}^{+0.36}$ & $43.47_{-20.98}^{+32.23}$ & $8.93_{-3.55}^{+3.45}$ & $29.42_{-14.69}^{+35.78}$ & $1.69_{-0.59}^{+0.65}$ & 8050 \\
\hline $0.005-0.22$ & $10.11_{-0.66}^{+0.51}$ & $60.76_{-24.1}^{+31.86}$ & $11.44_{-3.89}^{+5.59}$ & $23.73_{-10.8}^{+21.74}$ & $2.39_{-0.76}^{+1.08}$ & 209276 \\
\hline
\end{tabular}

Notes. The columns correspond to: (1) range of redshift considered; (2) median of the $\log \left(M_{\star} / M_{\odot}\right)$; (3) median of the $\mathrm{S} / \mathrm{N}$ (H $\alpha$ flux); (4) median of the S/N per whole spectrum range; (5) median of the $E W(\mathrm{H} \alpha)[\AA]$; (6) median of the Petrosian $R_{50}\left[{ }^{\prime \prime}\right]$; (7) number of galaxies.

\section{Empirical aperture correction and derivation of the SFR}

\subsection{Empirical aperture correction}

The fact that SDSS fibres ( $3^{\prime \prime}$ diameter) cover only a limited region of a galaxy at low-redshift Universe $(z<0.22)$, implies that only a limited amount of $\mathrm{H} \alpha$ emission can be measured. In order to derive the total $\mathrm{H} \alpha$ flux of each galaxy in our final sample, we corrected the SDSS $\mathrm{H} \alpha$ fluxes for aperture using the empirical aperture corrections in IP16 $6^{7}$. IP16 provide aperture corrections for emission lines and line ratios in a sample of spiral galaxies from the CALIFA database. Median growth curves of $\mathrm{H} \alpha$ and $\mathrm{H} \alpha / \mathrm{H} \beta$, up to $2.5 R_{50}$, were computed to simulate the effect of observing galaxies through apertures of varying radii. The median growth curve of the $\mathrm{H} \alpha$ flux (the $\mathrm{H} \alpha / \mathrm{H} \beta$ ratio) shows a monotonous increase (decrease) with radius, with no strong dependence on galaxy properties (i.e. inclination, morphological type, and stellar mass). The IP16 sample of CALIFA star-forming galaxies spans over the whole range in galaxy mass studied in this work (see IP16 for more details).

To strengthen the relevance of the CALIFA star-forming galaxies sample used in this work for the analysis of SDSS star-forming galaxies, a series of tests have been performed. First, the similarity between the galaxy stellar mass distributions of both samples has been statistically confirmed applying a Kolmogorov-Smirnov (KS) test, from which the following results have been obtained: $D_{\mathrm{n} 1, \mathrm{n} 2}=0.08$ and $p$-value $=0.36$, indicating that we cannot reject the hypothesis that both samples are drawn from the same distribution. Second, the relations between $S F R-M_{\star}$ and galaxy size- $M_{\star}$ for star-forming galaxies from CALIFA and SDSS samples have been analysed (see Appendix A); conclusive positive results have been achieved showing how the medians of the SFR of CALIFA galaxies are consistent with the results obtained in this work for SDSS galaxies over the whole range of galaxy mass (see Fig. A.1). Likewise for the galaxy size- $M_{\star}$ relation, the plot of $R_{50}$ vs. $M_{\star}$ (see Fig. A.2) shows that the medians of CALIFA galaxies are clearly consistent with the SDSS galaxies distribution.

\footnotetext{
7 For those galaxies in the final sample with $1.5 / R_{50} \geq 2.5$ (172 objects) aperture correction of the $\mathrm{H} \alpha$ flux was applied according to Fig. 2 in IP16.
}

SDSS $\mathrm{H} \alpha$ fluxes were previously corrected for extinction using the Balmer decrement as measured by the $\mathrm{H} \alpha / \mathrm{H} \beta$ ratio $\left(F_{\mathrm{H} \alpha / \mathrm{H} \beta}{ }^{\text {ap, corr }}\right)$. To do so, the median $\mathrm{H} \alpha / \mathrm{H} \beta$ flux ratio growth curve normalised to the $\mathrm{H} \alpha / \mathrm{H} \beta$ flux ratio at $2.5 R_{50}, X\left(\alpha \beta_{50}\right)$, was applied to each galaxy, and the $\mathrm{H} \alpha / \mathrm{H} \beta$ ratio was computed according to its corresponding value of $1.5 / R_{50}$ following IP16:

$F_{\mathrm{H} \alpha / \mathrm{H} \beta}{ }^{\text {ap, corr }}=\frac{F_{\mathrm{H} \alpha / \mathrm{H} \beta}{ }^{0}}{X\left(\alpha \beta_{50}\right)}$

being

$X\left(\alpha \beta_{50}\right)=0.0143 x^{5}-0.1091 x^{4}+0.2959 x^{3}-0.3125 x^{2}+$ $0.0274 x+1.1253$

where $x=\left(1.5 / R_{50}\right)$ for each galaxy, $F_{\mathrm{H} \alpha / \mathrm{H} \beta}{ }^{0}$ is the SDSS $\mathrm{H} \alpha / \mathrm{H} \beta$ ratio.

Theoretical case $\mathrm{B}$ recombination was assumed (the theoretical Balmer decrement, $I_{\mathrm{H} \alpha / \mathrm{H} \beta}=2.86 ; T=10^{4} \mathrm{~K}$, and low-density limit $n_{\mathrm{e}} \sim 10^{2} \mathrm{~cm}^{-3}$; Osterbrock 1989; Storey \& Hummer 1995) together with the Cardelli et al. (1989) extinction curve with $R_{\mathrm{v}}=A_{\mathrm{v}} / E(B-V)=3.1$ (O'Donnell 1994; Schlegel et al. 1998).

Equation (4) presents the aperture-corrected $\mathrm{H} \alpha$ flux, $F_{\mathrm{H} \alpha}^{\mathrm{ap} \text { corr }}$, as a function of the $\mathrm{H} \alpha$ flux in the SDSS fibre, $F_{\mathrm{H} \alpha}^{0}$, and the $X\left(\alpha_{50}\right)$, the median $\mathrm{H} \alpha$ flux growth curve normalised to the $\mathrm{H} \alpha$ flux at $2.5 R_{50}$ :

$F_{\mathrm{H} \alpha}^{\mathrm{ap}, \text { corr }}=\frac{F_{\mathrm{H} \alpha}^{0}}{X\left(\alpha_{50}\right)}$

\section{being}

$X\left(\alpha_{50}\right)=0.0037 x^{5}+0.0167 x^{4}-0.2276 x^{3}+0.5027 x^{2}+0.1599 x$ where $x=\left(1.5 / R_{50}\right)$ for each galaxy. According to IP16, for an aperture radius of $2.5 R_{50}$ an average of $\sim 85 \%$ of the total $\mathrm{H} \alpha$ flux of (non-AGN) spiral galaxies is enclosed.

Equation (5) presents the total $\mathrm{H} \alpha$ flux, $F_{\mathrm{H} \alpha}^{\mathrm{tot}}$, corrected for aperture effects and extinction:

$F_{\mathrm{H} \alpha}^{\mathrm{tot}}=\frac{F_{\mathrm{H} \alpha}^{\mathrm{ap}, \text { corr }}}{10^{-0.4 A(\mathrm{H} \alpha)}}$,

where the extinction in $\mathrm{H} \alpha, A(\mathrm{H} \alpha)=1.758 c(\mathrm{H} \beta)$. The reddening coefficient is $c(H \beta)=-\frac{1}{f(\mathrm{H} \alpha)} \log \left(\frac{F_{\mathrm{H} \alpha / \mathrm{H} \beta}^{\text {ap, corr }}}{I_{\mathrm{H} \alpha / \mathrm{H} \beta}}\right)$, and $f(\mathrm{H} \alpha)$ is the reddening curve. 


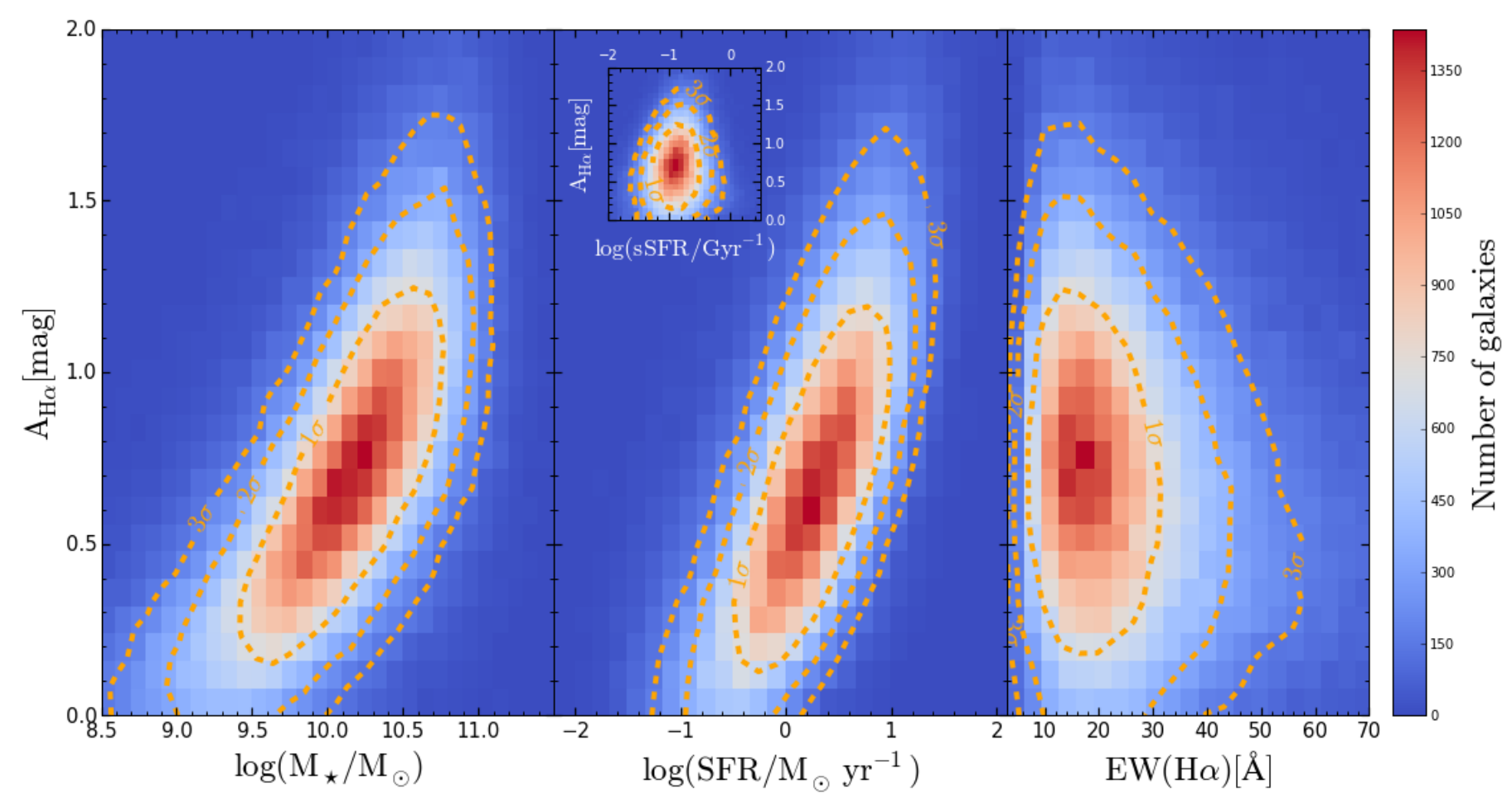

Fig. 2. Density plots for the SDSS star-forming galaxies: i) left panel: the relation between $\log \left(M_{\star} / M_{\odot}\right)$ and $A(\mathrm{H} \alpha)$; ii) central panel: the relation between $\log \left(S F R / M_{\odot} \mathrm{yr}^{-1}\right)$ and $A(\mathrm{H} \alpha)$, the inset plot shows the $A(\mathrm{H} \alpha)-\log \left(s S F R / \mathrm{Gyr}^{-1}\right)$ relation; iii) right panel: the relation between $E W(\mathrm{H} \alpha)$ and $A(\mathrm{H} \alpha)$. The dashed lines represent the $1 \sigma, 2 \sigma$, and $3 \sigma$ contours.

\subsection{Aperture-corrected star formation rate}

The present day SFR is defined as the stellar mass formed per unit time traced by the young stars. The SFR can be derived from the $\mathrm{H} \alpha$ luminosity, and it is parametrized as $S F R=L(\mathrm{H} \alpha) / \eta_{\mathrm{H} \alpha}$, the ratio of observed $\mathrm{H} \alpha$ luminosity ${ }^{8}$ to the conversion factor $\eta_{\mathrm{H} \alpha}$ (Brinchmann et al. 2004b). The parameter $\eta_{\mathrm{H} \alpha}$ varies with the physical properties of the galaxy, total stellar mass, and metallicity (see Charlot et al. 2002; Hirashita et al. 2003; Brinchmann et al. 2004b).

Kennicutt et al. (2009) assumed $\eta_{\mathrm{H} \alpha}$ is a constant value, $\eta_{\mathrm{H} \alpha}=10^{41.26} \mathrm{erg} / \mathrm{s} / M_{\odot} / \mathrm{yr}$, which resulted in a good typical conversion factor, though $\eta_{\mathrm{H} \alpha}$ can in fact vary as much as $\sim 0.4$ dex going from the least to the most massive galaxies (Brinchmann et al. 2004b). In order to parametrize the variation of the $\eta_{\mathrm{H} \alpha}$ as a function of stellar mass, we have used the median of the $\eta_{\mathrm{H} \alpha}$ likelihood distribution for the five stellar mass ranges as shown in Fig. 7 in Brinchmann et al. (2004b): $\log \left(M_{\star} / M_{\odot}\right)<8 ; 8<\log \left(M_{\star} / M_{\odot}\right)<9 ; 9<\log \left(M_{\star} / M_{\odot}\right)<10 ;$ $10<\log \left(M_{\star} / M_{\odot}\right)<11 ; \log \left(M_{\star} / M_{\odot}\right)>11$. The relation between $\eta_{\mathrm{H} \alpha}$ and galaxy stellar mass has been parametrized through a two-order polynomial fit as presented in Eq. (6):

$\log \left(\eta_{\mathrm{H} \alpha_{\mathrm{adj}}}\right)=-0.011 x^{2}+0.124 x+41.107$,

where $x=\log \left(M_{\star} / M_{\odot}\right)$.

We used the values of $\eta_{\mathrm{H} \alpha}$ from Eq. (6) to calculate the $S F R=L(\mathrm{H} \alpha) / \eta_{\mathrm{H} \alpha}$ for our galaxy sample. Hereafter, we refer to the aperture-corrected $\log (S F R)-\log \left(M_{\star}\right)$ relation as the $S F R$ $M_{\star}$ relation for our sample of galaxies. Also, from now on we

8 The $\mathrm{H} \alpha$ luminosity of a galaxy is $L(\mathrm{H} \alpha)=4 \pi d^{2} F_{\mathrm{H} \alpha}^{\text {tot }}, d$ being its luminosity distance corresponding to the SDSS spectroscopic redshift. refer to the SFR and the specific SFR $\left(s S F R=S F R / M_{\star}\right)$ derived here as the empirical aperture-corrected SFR and SSFR for star-forming galaxies.

Notwithstanding the above, possible effects associated with, for example, diffuse ionised gaseous emission, geometry, or galaxy inclination should be considered (e.g. Relaño et al. 2006; Kennicutt et al. 2009; Kennicutt \& Evans 2012; van der Wel et al. 2014). Although a comprehensive study of these effects could be hard to handle (e.g. Kennicutt \& Evans 2012), recent work by Iglesias-Páramo et al. $(2013,2016)$ concluded that the $\mathrm{H} \alpha$ flux growth curve and the $\mathrm{H} \alpha / \mathrm{H} \beta$ are insensitive to the galaxy inclination for a broad range of b/a (i.e. galaxy diameters ratio) including from edge-on to face-on galaxies. The effects of the geometry of the HII regions and the contribution of the diffuse ionised gas component are more difficult to quantify. We believe that these effects should be statistically minimised in this work, given the size of the sample and the large diversity of galaxy types used in IP16.

\section{Results}

\subsection{Extinction of the star-forming galaxies in the SDSS sample}

The extinction suffered by the $\mathrm{H} \alpha$ photons, $A(\mathrm{H} \alpha)$, derived in this work goes from 0 to $2 \mathrm{mag}$, with a median of $A(\mathrm{H} \alpha)=0.85 \mathrm{mag}^{9}$ (in agreement with previous work: Buat et al. 2002; Hopkins et al. 2003; Brinchmann et al. 2004b; Nakamura et al. 2004; Momcheva et al. 2013). $A(\mathrm{H} \alpha)$ presents a strong dependence with galaxy mass, as shown in Fig. 2

$9 \sim 4 \%$ of galaxies in our sample present negative values of $A(\mathrm{H} \alpha)$, though consistent with $A(\mathrm{H} \alpha)=0$ mag within the errors. For these galaxies the value of $A(\mathrm{H} \alpha)$ has been set to zero. 
(left panel), being larger for more massive galaxies, (see Brinchmann et al. 2004b; Whitaker et al. 2012; Momcheva et al. 2013; Koyama et al. 2015). Moreover, $A(\mathrm{H} \alpha)$ shows a trend with the SFR, with $A(\mathrm{H} \alpha) \leq 0.2$ mag for those galaxies presenting the lowest $\mathrm{SFR}\left(\log \left(S F R / M_{\odot} \mathrm{yr}^{-1}\right) \approx-0.5\right)$ whereas the highest values of $A(\mathrm{H} \alpha)$ are associated with the galaxies with the largest SFR (Fig. 2 central panel). Only a mild trend can be appreciated when $A(\mathrm{H} \alpha)$ is plotted versus the sSFR, confirming the strong weight that galaxy stellar mass has in the variation of $A(\mathrm{H} \alpha)$. The behaviour of $A(\mathrm{H} \alpha)$ as a function of $E W(\mathrm{H} \alpha)$ is shown in Fig. 2 (right panel). Galaxies showing the median value of $A(\mathrm{H} \alpha)$ cluster around $E W(\mathrm{H} \alpha) \sim 15 \AA$. For $E W(\mathrm{H} \alpha)>30 \AA, A(\mathrm{H} \alpha)$ presents a strong decline, approximating to values near $0.2 \mathrm{mag}$ and lower for $E W(\mathrm{H} \alpha)$ above $60 \AA$. An upper envelope for the maximum of the $A(\mathrm{H} \alpha)$ can be seen, decreasing towards larger values of $E W(\mathrm{H} \alpha)$.

The results presented above tell us that the $A(\mathrm{H} \alpha)$ extinction correction can be substantial and therefore must be applied to all galaxy samples to be used in the study of the SFR. Moreover, these results also show that the behaviour of $A(\mathrm{H} \alpha)$ appears to be different depending on the $E W(\mathrm{H} \alpha)$ of star-forming galaxies, showing a strong relation with galaxy mass and total SFR (see e.g. Koyama et al. 2015).

\subsection{Parametrizing the aperture-corrected $S F R-M_{\star}$ relation for star-forming galaxies}

In Fig. 3 we present the aperture-corrected $S F R-M_{\star}$ relation for our sample of star-forming galaxies (see Sect. 2.2). The SDSS fibre flux for each galaxy was corrected for aperture and extinction as explained in Sect. 3.1. The SFR was derived for each galaxy of the sample applying the methodology described in Sect. 3.2. The running median of the distribution of points plotted in Fig. 3 (red solid line) has been fitted with the following analytical expression:

$\log (\operatorname{SFR}(\mathrm{H} \alpha))=-0.03105 x^{3}+0.892 x^{2}-7.571 x+17.71$,

where $x=\log \left(M_{\star} / M_{\odot}\right)$.

For the sake of comparison with previous work (see Sect. 5), a straight linear fit to the running median distribution in Fig. 3 has been obtained as follows:

$\log (S F R)=\alpha x+\beta$,

where slope $\alpha=0.935( \pm 0.001), \beta=-9.208( \pm 0.001)$, and $x=\log \left(M_{\star} / M_{\odot}\right)$.

Figure 3 also shows the three-order polynomial fit to the running median of the values of SFR computed from the SDSS fibre flux without any correction by aperture (green dashed line). It is clear from Fig. 3 that the corrected SFR is, on average, more than 0.65 dex above the values corresponding to the SDSS fibre flux. The $S F R-M_{\star}$ relation obtained is consistent with the relation shown by Catalán-Torrecilla et al. (2015) for their sample of star-forming galaxies of the CALIFA survey (see Fig. A.1).

The inset plot shows the $s S F R-M_{\star}$ relation for our sample, corrected for aperture and extinction. The fits to the running medians of the aperture-corrected $s S F R-M_{\star}$ (red solid line) and the SDSS fibre fluxes (green dashed line) are also shown. From the $S S F R-M_{\star}$ relation, we observe that the fit to the running median distribution is decreasing slightly for the entire stellar mass range selected, consistent with recent predictions (see e.g. Sparre et al. 2015, and references therein).

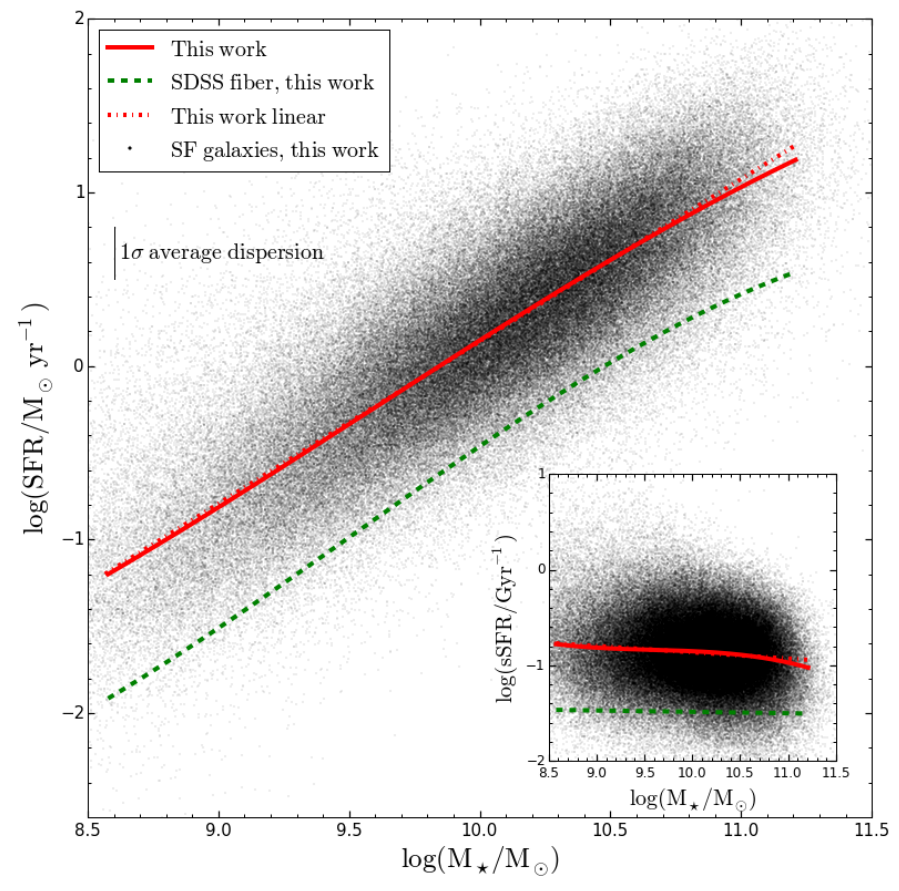

Fig. 3. Relation between the SFR and $M_{\star}$ for star-forming galaxies. The red solid line and green dashed line represent the fit to the running median for bins of 2000 objects in this work and in the SDSS fibre, respectively. The red dotted line represents the linear fit to the running median for bins of 2000 objects in this work. The vertical black line shows the $1 \sigma$ average dispersion $(\sim 0.3 \mathrm{dex})$. The inset plot shows the $s S F R-M_{\star}$ relation for our sample and the running median, for bins of 2000 objects, for the sSFR corrected for aperture (red solid line) and inside the SDSS fibre (green dashed line).

For the sake of completeness, in Table 2 we present median values $( \pm 1 \sigma$ confidence interval) of the derived $\log (S F R)$ for twelve stellar mass bins $\left(\Delta \log \left(M_{\star} / M_{\odot}\right)=0.25\right.$ dex each $)$ within the range $8.5 \leq \log \left(M_{\star} / M_{\odot}\right) \leq 11.5$. In Col. 1 the range of $\log \left(M_{\star} / M_{\odot}\right)$ is presented, Col. 2 shows the $\log \left(S F R / M_{\odot} \mathrm{yr}^{-1}\right)$, and the number of galaxies per stellar mass bin is quoted in Col. 3 .

\subsection{Aperture-corrected SFR as a function of the $M_{\star}$ and $z$ interval}

The aperture-corrected SFR as a function of the $M_{\star}$ is studied in six redshift bins in the range $0.005 \leq z \leq 0.22$. Figure 4 presents this evolution for the star-forming galaxy sample in the following redshift intervals: $0.005 \leq z<0.05,0.05 \leq z<0.08$, $0.08 \leq z<0.11,0.11 \leq z<0.14,0.14 \leq z<0.18$, and $0.18 \leq$ $z \leq 0.22$. In this figure, for each $z$ range, the corresponding line is the fit to the running median of the differences between the aperture-corrected SFR and the SFR within the SDSS fibre as a function of the $M_{\star}$.

This figure shows that: i) over the whole range of galaxy stellar masses studied, the average aperture correction goes from $\sim 0.7$ to $\sim 0.8$ for $0.005 \leq z \leq 0.05$; ii) aperture corrections increase with galaxy mass for each redshift interval for $0.05 \leq$ $z \leq 0.22$; reaching from $\sim 0.2$ for $\log \left(M_{\star} / M_{\odot}\right)=10$ to $\sim 0.6$ for $\log \left(M_{\star} / M_{\odot}\right)=11$, for $0.18 \leq z \leq 0.22$.

In Table 3, for each $z$ range, we present the median $\left( \pm 1 \sigma\right.$ confidence interval) of the derived $\log \left(S F R / M_{\odot} \mathrm{yr}^{-1}\right)$ and $\log \left(M_{\star} / M_{\odot}\right)$ along three stellar mass bins and for the whole mass range $\left(8.5 \leq \log \left(M_{\star} / M_{\odot}\right) \leq 11.5\right)$ for six redshift bins 
Table 2. Values of aperture-corrected SFR corresponding to the median $( \pm 1 \sigma$ confidence interval) of the distribution for the star-forming galaxies in twelve stellar mass bins in the total sample.

\begin{tabular}{ccc}
\hline \hline$(1)$ & $(2)$ & $(3)$ \\
$\Delta \log \left(M_{\star} / M_{\odot}\right)$ & $\log \left(S F R / M_{\odot} \mathrm{yr}^{-1}\right)$ & \# galaxies \\
\hline $8.50-8.75$ & $-1.11_{-0.42}^{+0.56}$ & 3926 \\
$8.75-9.00$ & $-0.94_{-0.40}^{+0.49}$ & 6724 \\
$9.00-9.25$ & $-0.69_{-0.40}^{+0.43}$ & 10397 \\
$9.25-9.50$ & $-0.45_{-0.38}^{+0.41}$ & 15405 \\
$9.50-9.75$ & $-0.19_{-0.36}^{+0.38}$ & 22512 \\
$9.75-10.00$ & $0.03_{-0.34}^{+0.36}$ & 29989 \\
$10.00-10.25$ & $0.26_{-0.34}^{+0.35}$ & 35854 \\
$10.25-10.50$ & $0.49_{-0.35}^{+0.33}$ & 36230 \\
$10.50-10.75$ & $0.71_{-0.35}^{+0.33}$ & 28016 \\
$10.75-11.00$ & $0.92_{-0.33}^{+0.33}$ & 14986 \\
$11.00-11.25$ & $1.08_{-0.37}^{+0.33}$ & 4563 \\
$11.25-11.50$ & $1.19_{-0.40}^{+0.38}$ & 674 \\
\hline $8.50-11.50$ & $0.23_{-0.69}^{+0.58}$ & 209276 \\
\hline
\end{tabular}

Notes. The columns correspond to: (1) range of $\log \left(M_{\star} / M_{\odot}\right)$; (2) median value of $\log \left(S F R / M_{\odot} \mathrm{yr}^{-1}\right)$ and error value; (3) number of galaxies.

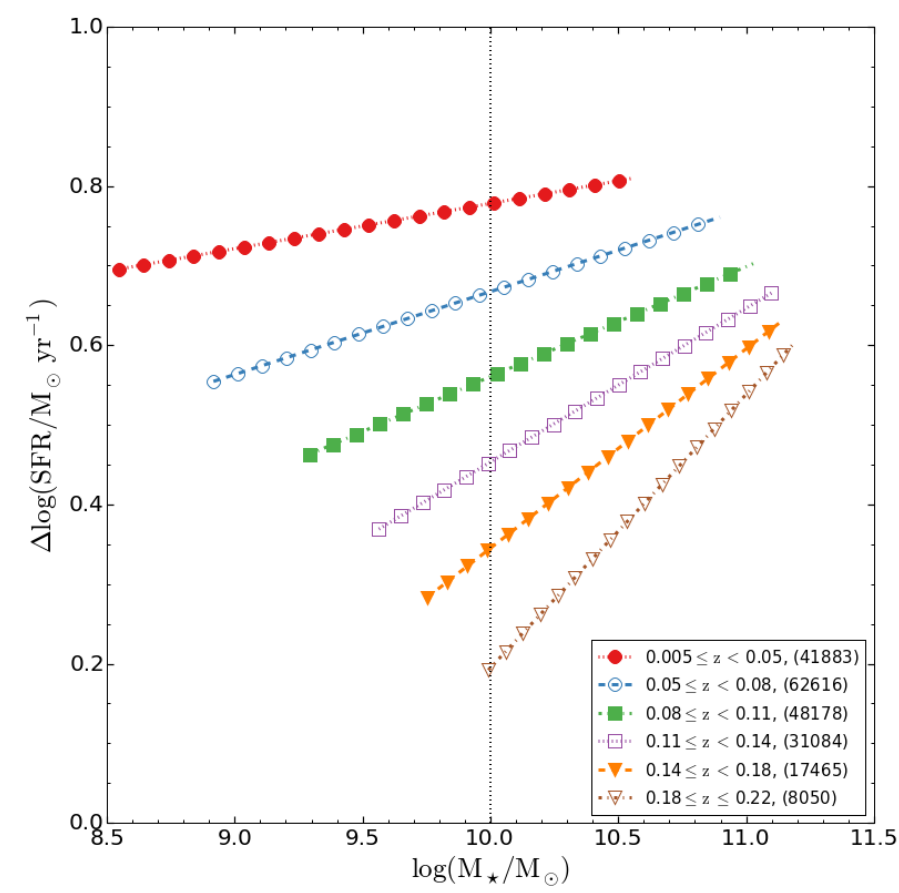

Fig. 4. Empirical SFR corrections (total - SDSS fibre) vs. $M_{\star}$ relation. Red, blue, green, magenta, orange, and gold lines represent the fit to the running median for bins of 1000 objects in six redshift bins up to $z=0.22$ for a sample of star-forming galaxies. The numbers of starforming galaxies in each redshift bin appear on the legend. The vertical black dotted line corresponds to the reference value at $\log \left(M_{\star} / M_{\odot}\right)=$ 10 .

in the range $0.005 \leq z \leq 0.22$. In Col. 1 the $\Delta z$ is displayed, the $\Delta \log \left(M_{\star} / M_{\odot}\right)$ is presented in Col. 2, Col. 3 shows the $\log \left(S F R / M_{\odot} \mathrm{yr}^{-1}\right)$, Col. 4 shows the $\log \left(M_{\star} / M_{\odot}\right)$, and the number of galaxies per stellar mass bin is quoted in Col. 5. All this information is presented in Fig. 5 for the sample of galaxies in
Table 3. Values of stellar mass and aperture-corrected SFR per stellar mass and redshift bins corresponding to the median $( \pm 1 \sigma$ confidence interval) of the distribution for the star-forming galaxies in the total sample.

\begin{tabular}{|c|c|c|c|c|}
\hline (1) & (2) & (3) & (4) & (5) \\
\hline$\Delta z$ & $\Delta \log \left(M_{\star}\right)$ & $\log (S F R)$ & $\log \left(M_{\star}\right)$ & \# galaxies \\
\hline \multirow{4}{*}{$0.005-0.05$} & $8.50-9.50$ & $-0.79_{-0.45}^{+0.42}$ & $9.10_{-0.33}^{+0.27}$ & 25872 \\
\hline & $9.50-10.50$ & $-0.14_{-0.42}^{+0.44}$ & $9.82_{-0.23}^{+0.34}$ & 15354 \\
\hline & $10.50-11.50$ & $0.63_{-0.47}^{+0.39}$ & $10.62_{-0.10}^{+0.16}$ & 657 \\
\hline & $8.50-11.50$ & $-0.55_{-0.54}^{+0.58}$ & $9.34_{-0.46}^{+0.56}$ & 41883 \\
\hline \multirow{4}{*}{$0.05-0.08$} & $8.50-9.50$ & $-0.35_{-0.39}^{+0.37}$ & $9.35_{-0.22}^{+0.11}$ & 8775 \\
\hline & $9.50-10.50$ & $0.04_{-0.37}^{+0.39}$ & $9.96_{-0.29}^{+0.32}$ & 48229 \\
\hline & $10.50-11.50$ & $0.64_{-0.38}^{+0.37}$ & $10.64_{-0.10}^{+0.18}$ & 5612 \\
\hline & $8.50-11.50$ & $0.03_{-0.42}^{+0.45}$ & $9.93_{-0.40}^{+0.43}$ & 62616 \\
\hline \multirow{4}{*}{$0.08-0.11$} & $8.50-9.50$ & $-0.04_{-0.57}^{+0.42}$ & $9.34_{-0.22}^{+0.11}$ & 1265 \\
\hline & $9.50-10.50$ & $0.29_{-0.34}^{+0.33}$ & $10.16_{-0.29}^{+0.22}$ & 35641 \\
\hline & $10.50-11.50$ & $0.67_{-0.36}^{+0.36}$ & $10.66_{-0.12}^{+0.20}$ & 11272 \\
\hline & $8.50-11.50$ & $0.36_{-0.37}^{+0.39}$ & $10.25_{-0.36}^{+0.34}$ & 48178 \\
\hline \multirow{4}{*}{$0.11-0.14$} & $8.50-9.50$ & $0.22_{-0.50}^{+0.45}$ & $9.38_{-0.18}^{+0.09}$ & 351 \\
\hline & $9.50-10.50$ & $0.53_{-0.37}^{+0.29}$ & $10.28_{-0.31}^{+0.16}$ & 16143 \\
\hline & $10.50-11.50$ & $0.78_{-0.33}^{+0.33}$ & $10.69_{-0.14}^{+0.21}$ & 14590 \\
\hline & $8.50-11.50$ & $0.64_{-0.37}^{+0.34}$ & $10.48_{-0.36}^{+0.30}$ & 31084 \\
\hline \multirow{4}{*}{$0.14-0.18$} & $8.50-9.50$ & $0.60_{-0.55}^{+0.37}$ & $9.40_{-0.15}^{+0.07}$ & 136 \\
\hline & $9.50-10.50$ & $0.63_{-0.45}^{+0.36}$ & $10.23_{-0.30}^{+0.20}$ & 6292 \\
\hline & $10.50-11.50$ & $0.95_{-0.31}^{+0.31}$ & $10.77_{-0.17}^{+0.21}$ & 11037 \\
\hline & $8.50-11.50$ & $0.85_{-0.41}^{+0.34}$ & $10.62_{-0.44}^{+0.28}$ & 17465 \\
\hline \multirow{4}{*}{$0.18-0.22$} & $8.50-9.50$ & $1.06_{-0.68}^{+0.24}$ & $9.42_{-0.13}^{+0.07}$ & 53 \\
\hline & $9.50-10.50$ & $0.69_{-0.40}^{+0.39}$ & $10.25_{-0.25}^{+0.17}$ & 2931 \\
\hline & $10.50-11.50$ & $1.12_{-0.34}^{+0.31}$ & $10.90_{-0.25}^{+0.23}$ & 5066 \\
\hline & $8.50-11.50$ & $0.99_{-0.46}^{+0.36}$ & $10.70_{-0.50}^{+0.36}$ & 8050 \\
\hline
\end{tabular}

Notes. The columns correspond to: (1) range of $z$ considered; (2) range of $\log \left(M_{\star} / M_{\odot}\right)$; (3) median value of the $\log \left(S F R / M_{\odot} \mathrm{yr}^{-1}\right)$ and error value; (4) median value of the $\log \left(M_{\star} / M_{\odot}\right)$ and error value; (5) number of galaxies.

the range of redshift and stellar mass considered. In this figure we show the relation between total SFR and $M_{\star}$ for our sample of star-forming galaxies, along three stellar mass bins for the whole mass range $\left(8.5 \leq \log \left(M_{\star} / M_{\odot}\right) \leq 11.5\right)$, and for six redshift bins in the range $0.005 \leq z \leq 0.22$.

\section{Discussion}

We start by comparing our SFR values (Sect. 3.2) with the ones provided by the MPA-JHU database ${ }^{10}$. The MPA-JHU database gives the SFR within the $3^{\prime \prime}$ fibre of the SDSS and the total SFR corrected for aperture (Brinchmann et al. 2004b; Salim et al. 2007) (hereafter $S F R_{\mathrm{MPA}}$ ). Brinchmann et al. (2004b) originally corrected fibre SFRs from aperture effects using the resolved colour information available for each galaxy. Salim et al. (2007)

\footnotetext{
${ }^{10}$ A total of 4566 star-forming galaxies from our final sample are not considered since the MPA-JHU database provided -9999 for their SFR values.
} 


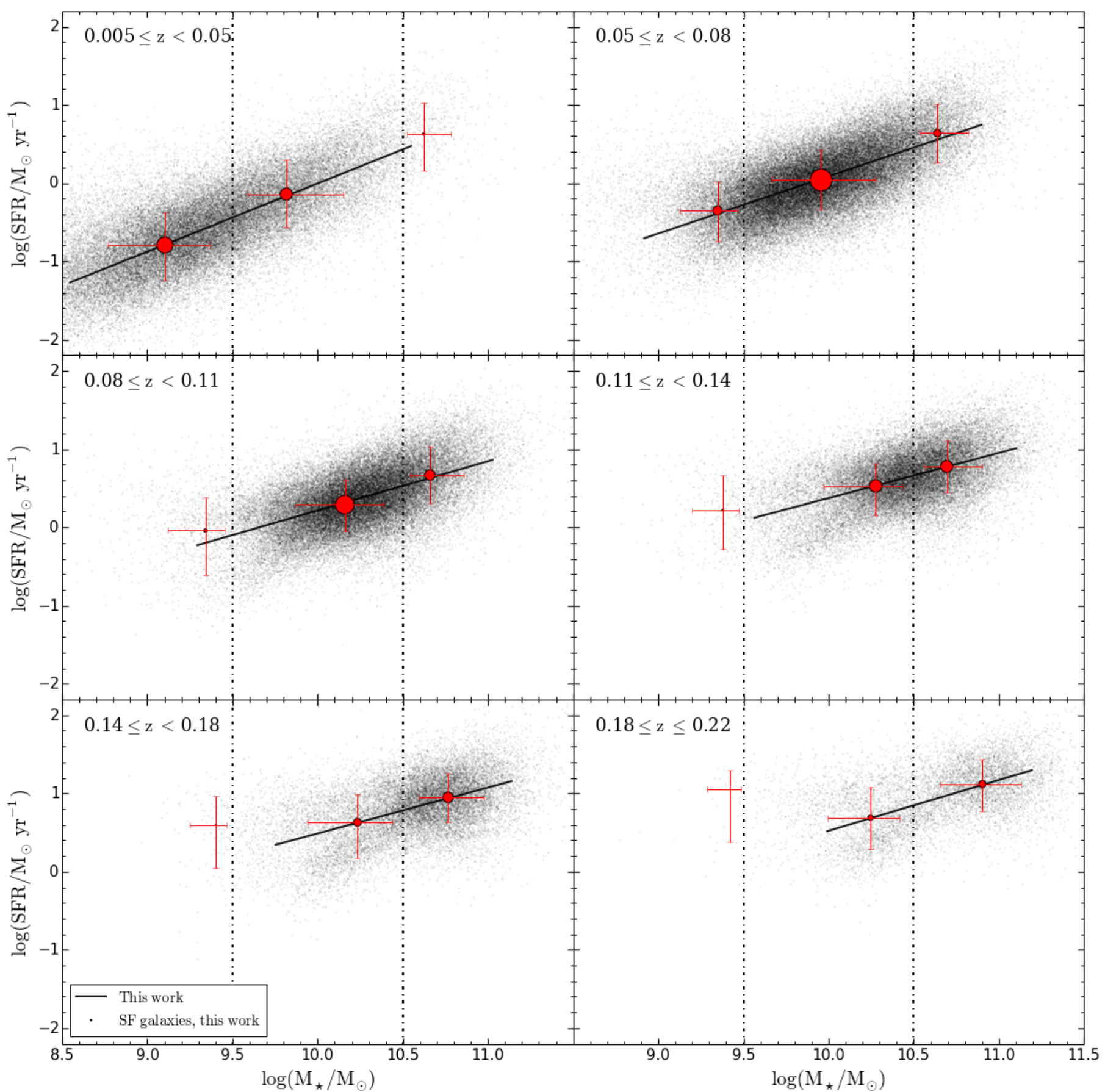

Fig. 5. Relation between the SFR and $M_{\star}$ for SDSS star-forming galaxies, along three stellar mass bins for the whole mass range $(8.5 \leq$ $\left.\log \left(M_{\star} / M_{\odot}\right) \leq 11.5\right)$ and for six redshift ranges in the range $0.005 \leq z \leq 0.22$. The black solid line represents the fit to the running median for bins of 1000 objects in this work for each redshift range. Red dots represent the medians of SFR and $M_{\star}$ per stellar mass bin and redshift range. The error bars in $x$ - and $y$-axis represent the $\pm 1 \sigma$ confidence interval for stellar mass and SFR, respectively. The symbol sizes increase with the number of objects contained in each mass range.

noted an overestimation of these SFRs for galaxies with low levels of star formation, attributed to the larger contribution from dusty high-metallicity starbursts inside these galaxies ${ }^{11}$. Consequently, these authors improved the Brinchmann et al. (2004b) technique (see Salim et al. 2007, for a detailed description) and several studies have used these values of SFR reported by these authors (e.g. Zahid et al. 2012; Renzini \& Peng 2015).

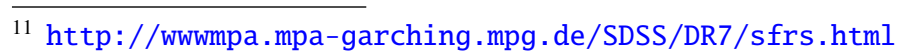

A different aperture correction was applied by Hopkins et al. (2003), assuming that the $\mathrm{H} \alpha$ emission-line flux can be traced across the whole galaxy by the $r$-band emission. Hopkins et al. (2003) derived the total $\mathrm{H} \alpha$ flux as a function of the difference between the total petrosian $r$-band magnitude $\left(r_{\text {petro }}\right)$ and the corresponding magnitude inside the SDSS fibre $\left(r_{\text {fibre }}\right)$ as: $F_{\mathrm{H} \alpha}^{\text {corr }}=F_{\mathrm{H} \alpha}^{0} \times 10^{-0.4\left(r_{\text {petro }}-r_{\text {fibre }}\right)}$ (see also Pilyugin et al. 2013). For the sake of comparison, the total $\mathrm{H} \alpha$ flux and the corresponding $L(\mathrm{H} \alpha)$ and SFR for all the galaxies in our final sample was recalculated according to the aperture correction recipe 


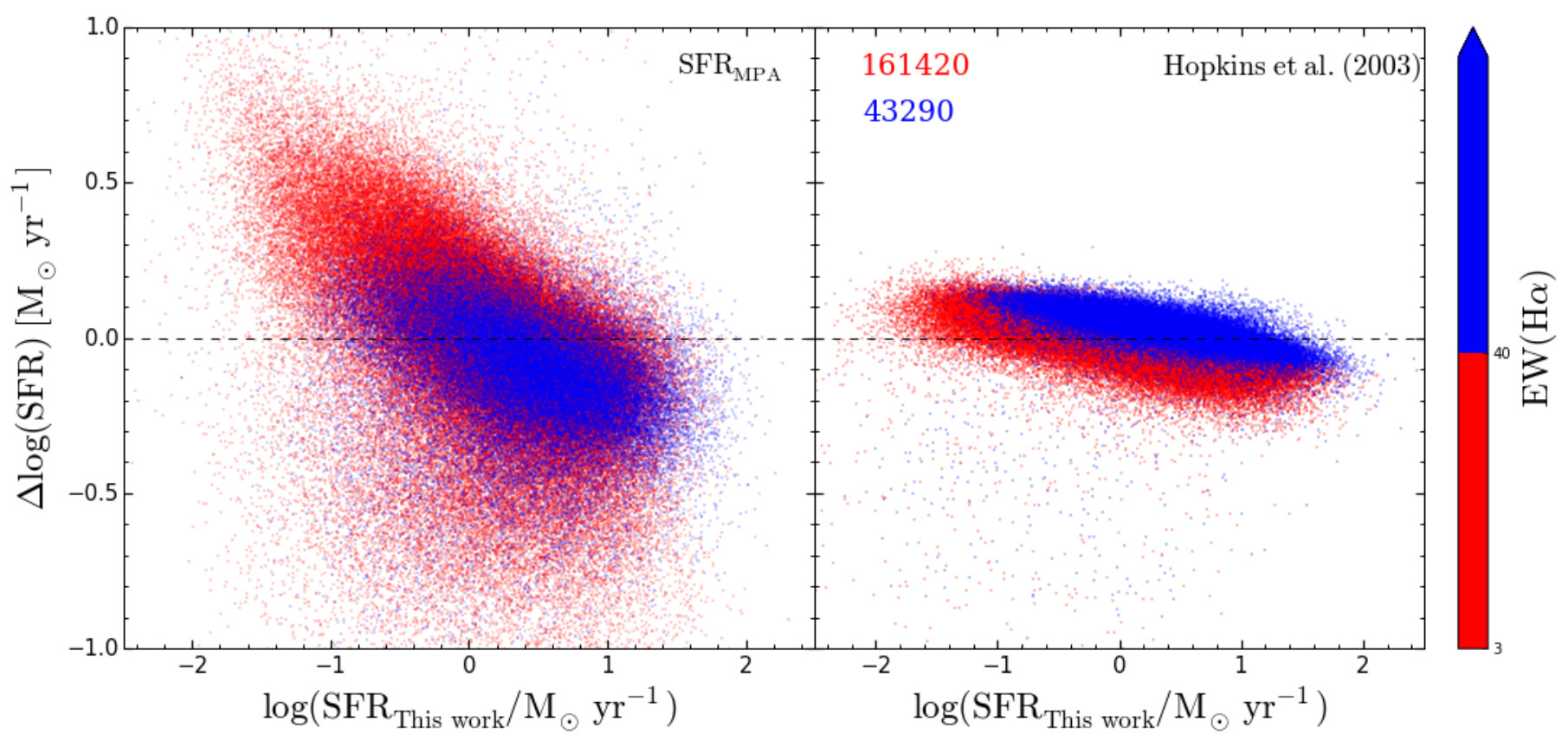

Fig. 6. Left panel: difference, $\Delta \log (S F R)$, of total SFR provided by MPA-JHU (Brinchmann et al. 2004b; Salim et al. 2007) and our total empirical $S F R_{\text {This work }}$ as a function of $S F R_{\text {This work }}$; right panel: difference of the total SFR derived using the Hopkins et al. (2003) aperture correction for

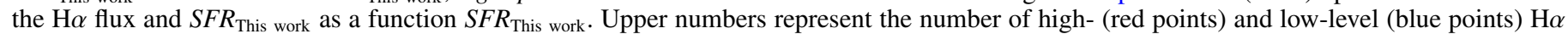
emitting galaxies (colour-coded according to the $E W(\mathrm{H} \alpha)$ colour bar). The black dashed line indicates $\Delta \log (S F R)=0$.

from Hopkins et al. (2003) (Eq. (B3)). Here we have refined the Hopkins et al. (2003) recipe for $\mathrm{H} \alpha$ flux aperture correction following the methodology explained in Sect. 3.2. The only difference between our refined Hopkins et al. (2003) recipe and Hopkins et al. (2003) methodology is that the former is based on Eq. (6) which converts $\mathrm{H} \alpha$ into SFR, while the latter uses a constant conversion value of 41.26 .

In Fig. 6 (left plot) we show the difference between $S F R_{\mathrm{MPA}}$ and the empirical SFR derived in this work $\left(S F R_{\text {This work }}\right)$ as a

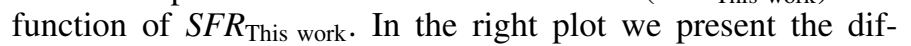
ference between the SFR derived applying the Hopkins et al. (2003) aperture correction and $S F R_{\text {This work }}$ as a function of $S F R_{\text {This work }}$. We discriminate between high- $(E W(\mathrm{H} \alpha) \geq 40 \AA$; blue points) and low-level $(E W(\mathrm{H} \alpha)<40 \AA$; red points $) \mathrm{H} \alpha$ emitting galaxies. The left plot shows that: i) a significant scatter is present, especially for the low-level $\mathrm{H} \alpha$ emitters, being critical (over $1 \mathrm{dex}$ ) for low SFRs; and ii) those galaxies with large SFRs and high $E W(\mathrm{H} \alpha)$ show values of $S F R_{\mathrm{MPA}}$ somewhat closer to the ones derived in this work, though large systematic differences and big scatter remain. In the right plot, the average SFR values derived according to Hopkins et al. (2003) show a systematic difference with respect to $S F R_{\text {This work }}$ ones, and a significant scatter (up to $\sim 1 \mathrm{dex}$ ) is apparent. Galaxies with $E W(\mathrm{H} \alpha)<40 \AA$ present a larger scatter and a clear offset from those with $E W(\mathrm{H} \alpha) \geq 40 \AA$. The differences between the SFR values derived using Hopkins et al. (2003) recipe and the ones obtained in this work could be reduced when we refine the Hopkins et al. (2003) methodology, as explained above; however, a noticeable scatter and some systematics between red and blue points remain. In this regard, we should bear in mind that the $r$-band flux of star-forming galaxies includes a contribution from $\mathrm{H} \alpha$ and nearby emission lines and, in fact, should behave as a rough tracer of HII regions. On the other hand, we note in passing that those objects with prominent bulges (likely correlating with the lower level star-formation objects in this sample) should contribute to the emission in the $r$-band in a more significant manner (e.g. Richards et al. 2016).

In Fig. 7 we show the $S F R_{\text {This work }}$ versus $M_{\star}$ for each galaxy in our sample and compare the fit to the $S F R-M_{\star}$ relation derived with previous results from theoretical and observational works. We present (panel a) the linear fit to the running median to the $S F R-M_{\star}$ relation: i) using the empirical total SFR from this work; ii) based on the $S F R_{\mathrm{MPA}}$; iii) SFR that we obtained applying the Hopkins et al. (2003) recipe and also our refined method of Hopkins et al. (2003) recipe; iv) SFR for the $\mathrm{H} \alpha$ SDSS fibre flux derived in this work. Finally, in this figure (panel c) we also show recent theoretical predictions for the SFR vs. $M_{\star}$ relation from i) Sparre et al. (2015) at $z=0$ using the Illustris simulation for star-forming galaxies with stellar masses between $10^{9} M_{\odot}$ and $10^{10.5} M_{\odot}$; and ii) by Dutton et al. (2010) at $z=0$ using a semi-analytic model for star-forming galaxies with stellar masses between $10^{9} M_{\odot}$ and $10^{11} M_{\odot}$. In Fig. 7 (panels b and d) we present the differences between the fit to the $S F R-M_{\star}$ relation of this work and those obtained for MPAJHU, Hopkins et al. (2003), our refined method of Hopkins et al. (2003) recipe, Dutton et al. (2010), and Sparre et al. (2015). The difference between the aperture-corrected SFR in this work and the SFR corresponding to the SDSS fibre is also shown.

As we can see in Fig. 7, the overall difference between $S F R_{\mathrm{MPA}}$ and this work amounts to $\sim 0.6$ dex going from the less to the more massive galaxies. The fit to the $S F R-M_{\star}$ relation shows that $S F R_{\mathrm{MPA}}$ leads to systematically larger values of the SFR for masses $M_{\star} / M_{\odot}<10^{9}(\approx 0.35 \mathrm{dex})$ and conversely, under-predicts the $\mathrm{SFR}^{12}$ by $\approx 0.3 \mathrm{dex}$ for $M_{\star} / M_{\odot}>10^{11}$.

12 We have checked that the difference between $S F R_{\mathrm{MPA}}$ and our SFR values is systematic along the range $0.005 \leq z \leq 0.22$. In the larger redshift interval $(0.11 \leq z \leq 0.22)$ the SFR predictions by different methods converge as expected, since the geometrical region covered by the SDSS fibre increases. 


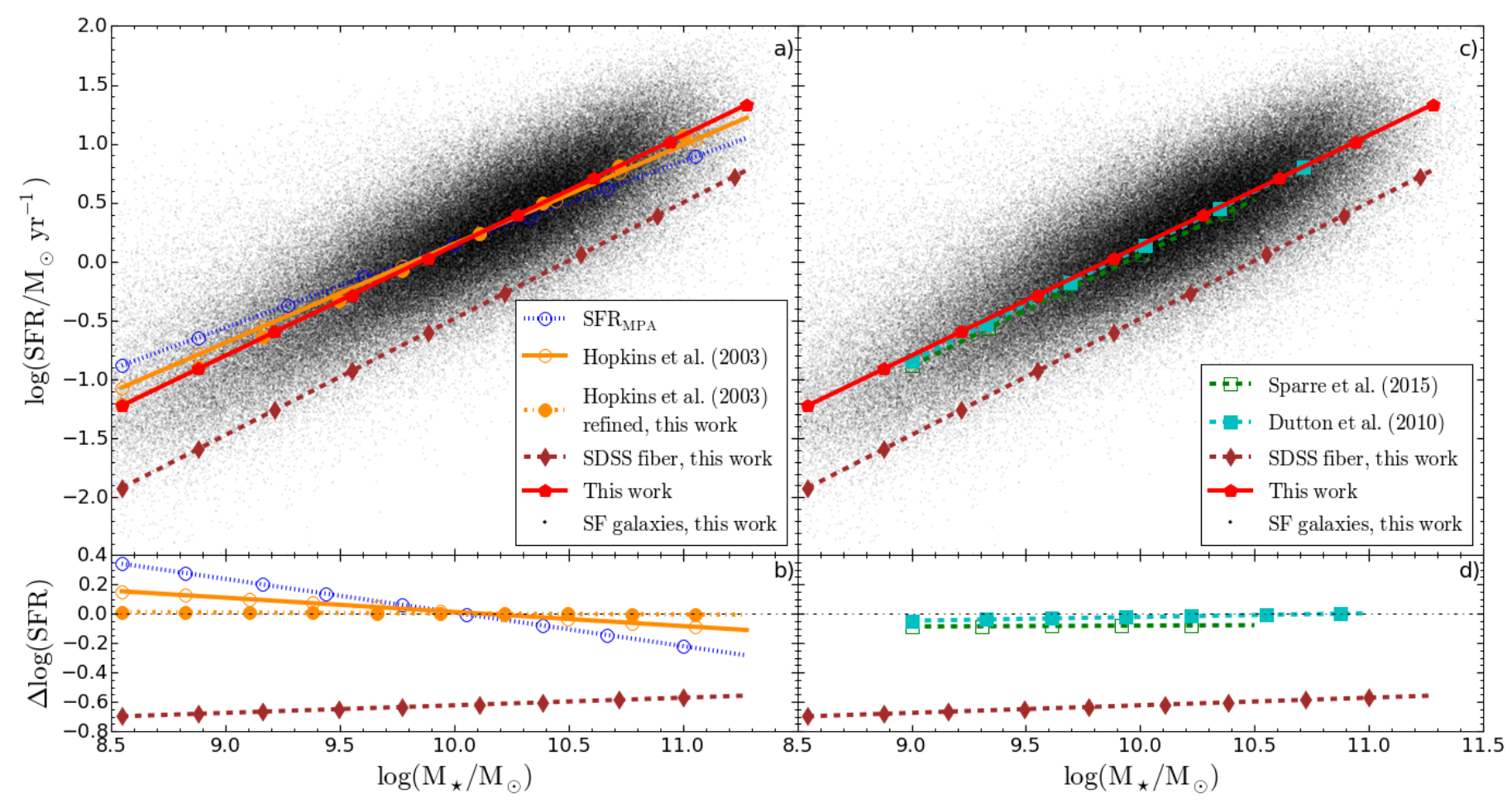

Fig. 7. $S F R_{\text {This work }}-M_{\star}$ relation for star-forming galaxies compared with previous theoretical and observational works. Comparison with observational studies: Panel a) $S F R-M_{\star}$ fits to the running median for bins of 2000 objects obtained in this work (red solid line) and from the SDSS fibre (brown dashed line), together with the values provided by MPA-JHU (blue dotted line), using the Hopkins et al. (2003) recipe (orange solid line), and from our refined method of Hopkins et al. (2003) recipe (orange dashed line). Panel b) Difference vs. $M_{\star}$ between SDSS fiber SFRs and $S F R_{\text {This work }}$ and between observational studies and $S F R_{\text {This work }}$ (colours as in upper left panel). Dotted line shows $\Delta \log (S F R)=0$. Comparison with theoretical studies: Panel c) $S F R-M_{\star}$ fits to the running median for bins of 2000 objects obtained in this work (red solid line) and from the SDSS fibre (brown dashed line), together with the predictions from Sparre et al. (2015) (green dashed line) and Dutton et al. (2010; cyan dashed line). Panel d) Difference vs. $M_{\star}$ between SDSS fiber SFRs and $S F R_{\text {This work }}$ and between theoretical predictions and $S F R_{\text {This work }}$ (colours as in upper right panel $)$. Dotted line shows $\Delta \log (S F R)=0$.

Recent studies have reached similar results (e.g. Richards et al. $2016)^{13}$. In this respect, we should bear in mind that galaxies with $\log \left(M_{\star} / M_{\odot}\right) \gtrsim 10.5$ may host large bulges containing little star formation, and therefore for these objects aperture correction can be problematic (e.g. Brinchmann et al. 2004b; Momcheva et al. 2013; Richards et al. 2016). Conversely, for less massive galaxies, the aperture problems become considerably smaller. The empirical $S F R-M_{\star}$ fit of this work also shows a systematic difference with the relation derived using Hopkins et al. (2003). The fit to the points obtained with the refined method of the Hopkins et al. (2003) recipe presented here gives a much better result. Excellent agreement is found with the Sparre et al. (2015) and Dutton et al. (2010) predictions.

It is important to note that the MS slope obtained from $S F R_{\mathrm{MPA}}$ is $\sim 0.71$. As far as we know, those studies that used the SFR values from the MPA-JHU catalogue obtained similar slopes (e.g. Elbaz et al. 2007; Zahid et al. 2012; Renzini \& Peng 2015 , show slopes of $0.77,0.71,0.76$, respectively). On the other hand, as mentioned in Sect. 4.2 (see Eq. (8)), the MS slope obtained in this work is 0.935 . The MS slope calculated in this work is in very good agreement with the predictions of semi-analytical models by Dutton et al. (2010), giving a zero point of the MS relation consistent with our result. Agreement is also found after the comparison of the MS slope with the predictions of the Illustris cosmological hydrodynamical simulations of galaxy formation by Sparre et al. (2015).

\footnotetext{
${ }^{13}$ We note that in Richards et al. (2016) the sample of galaxies with
} $\log \left(S F R / M_{\odot} \mathrm{yr}^{-1}\right)<-1.5$ seems to be underpopulated.
Finally, the $S F R-M_{\star}$ relation obtained in this work appears consistent with recent IFS observations of modest-size samples of galaxies (e.g. Catalán-Torrecilla et al. 2015; Richards et al. 2016). This concordance is relevant given the fact that total SFR values by Catalán-Torrecilla et al. (2015) and Richards et al. (2016) are obtained integrating the $\mathrm{H} \alpha$ flux over the spatially resolved galaxies, whereas in this work total SFRs are derived from the SDSS fibre $\mathrm{H} \alpha$ flux corrected for aperture effects.

\section{Summary and conclusions}

This work provides a robust study of the total empirical SFR of galaxies and its dependence on stellar mass, extinction, and redshift. Here we present the first study that uses total $\mathrm{H} \alpha$ flux, corrected for aperture from empirical $\mathrm{H} \alpha$ growth curves, to analyse the behaviour of present-day SFR and SSFR of all SDSS starforming galaxies. This empirical aperture correction is based on a sample of 165 spiral galaxies from the CALIFA project (IP16). Concurrently, we have derived the $S F R-M_{\star}$ and the $\mathrm{S} S F R-M_{\star}$ relations applying our considerations. We have compared these relations, free from aperture effects, with other methods (e.g. Hopkins et al. 2003; Brinchmann et al. 2004b) and with predictions from recent theoretical models (e.g. Dutton et al. 2010; Sparre et al. 2015).

Our main conclusions are the following:

i) The mean empirical aperture-corrected SFR, averaged over galaxy stellar mass, for the entire sample of SDSS starforming galaxies amounts to $\sim 0.65$ dex. 
ii) The average aperture-corrected SFR for nearby galaxies, $0.005 \leq z \leq 0.05$, is between $\sim 0.7$ and $\sim 0.8$. For larger $z(0.05 \leq z \leq 0.22)$ aperture corrections increase with galaxy mass along each redshift interval.

iii) The aperture-free $S F R-M_{\star}$ relation obtained in this work is: $\log (S F R)=0.935( \pm 0.001) \log \left(M_{\star} / M_{\odot}\right)-9.208( \pm 0.001)$. When comparing total SFRs from previous works with ours, we find: a) the $S F R-M_{\star}$ relation by MPA-JHU (Brinchmann et al. 2004b; Salim et al. 2007) provides larger SFR values (by $\sim 0.3 \mathrm{dex}$ ) for $\log \left(M_{\star} / M_{\odot}\right) \leq 9$; conversely, MPA-JHU SFR values for $\log \left(M_{\star} / M_{\odot}\right) \geq 11$ appear systematically lower (by up to $0.3 \mathrm{dex}$ ); b) overall consistency is found with selected observational studies based on integral field spectroscopy of individual galaxies (e.g. Catalán-Torrecilla et al. 2015; Richards et al. 2016); c) excellent agreement is obtained with theoretical predictions of recent semi-analytic models of disc galaxies (Dutton et al. 2010), and with Illustris hydrodynamical simulations (Sparre et al. 2015); d) the SFRs derived applying the Hopkins et al. (2003) recipe show a systematic difference along the range of galaxy stellar mass. When this derivation is refined following the methodology described in this work (see Sect. 3.2), together with the Hopkins et al. (2003) formula for $\mathrm{H} \alpha$ flux aperture correction, the SFRs obtained appear consistent with our results for high SFR objects, showing substantial scatter notably for the lowest SFR values.

iv) A slope $\mathrm{d} \log (S F R) / \mathrm{d} \log \left(M_{\star}\right)=0.935$ is derived for our $S F R-M_{\star}$ relation. This value is higher than those found with MPA-JHU data (e.g. Elbaz et al. 2007; Renzini \& Peng 2015 ), which are $\sim 0.76$, with a significant spread. The slope found in this work perfectly agrees with recent theoretical predictions (Dutton et al. 2010; Sparre et al. 2015), giving further support to the empirical $\mathrm{H} \alpha$ aperture correction used. For the specific SFR (sSFR) a slightly decreasing trend is seen along the entire range of stellar mass explored.

v) The total SFR values of the entire sample present a clear correlation with extinction, in overall qualitative agreement with recent works (e.g. Whitaker et al. 2012; Koyama et al. 2015).

Acknowledgements. We are grateful to Simon Verley, Maria del Carmen Argudo Fernandez, and Cristina Catalan Torrecilla for useful discussions. We thank the anonymous referee for very constructive suggestions that have improved this manuscript. SDP, JVM, JIP, CK, and EPM acknowledge financial support from the Spanish Ministerio de Economía y Competitividad under grant AYA201347742-C4-1-P, and from Junta de Andalucía Excellence Project PEX2011FQM-7058. FFRO acknowledges the exchange programme Study of EmissionLine Galaxies with Integral-Field Spectroscopy (SELGIFS, FP7-PEOPLE-2013IRSES-612701), funded by the EU through the IRSES scheme.

Funding for SDSS-III has been provided by the Alfred P. Sloan Foundation, the Participating Institutions, the National Science Foundation, and the U.S. Department of Energy Office of Science. SDSS-III is managed by the Astrophysical Research Consortium for the Participating Institutions of the SDSS III Collaboration including the University of Arizona, the Brazilian Participation Group, Brookhaven National Laboratory, Carnegie Mellon University, University of Florida, the French Participation Group, the German Participation Group, Harvard University, the Instituto de Astrofísica de Canarias, the Michigan State/Notre Dame/JINA Participation Group, Johns Hopkins University, Lawrence Berkeley National Laboratory, Max Planck Institute for Astrophysics, Max Planck Institute for Extraterrestrial Physics, New Mexico State University, New York University, Ohio State University, Pennsylvania State University, University of Portsmouth, Princeton University, the Spanish Participation Group, University of Tokyo, University of Utah, Vanderbilt University, University of Virginia, University of Washington, and Yale University. The SDSS-III web site is http://www.sdss3.org/. This study makes use of the results based on the Calar Alto Legacy Integral Field Area (CAL IFA) survey (http://califa.caha.es/) performed at Calar Alto observatory. This research made use of Python (http://www.python.org) and IPython
(Pérez \& Granger 2007); Numpy (Van Der Walt et al. 2011); Scipy (Jones et al. 2001); Pandas (McKinney 2010); of Matplotlib (Hunter 2007), a suite of opensource python modules that provides a framework for creating scientific plots. We also acknowledge the use of astroML (Vanderplas et al. 2012; Ivezić et al. 2014), a Python module for machine learning and data mining built on numpy, scipy, scikit-learn, and matplotlib, and distributed under the 3-clause BSD licenseastroML. The astroML web is http://www. astroml.org. This research made use of Astropy, a community-developed core Python package for Astronomy (Astropy Collaboration et al. 2013). The Astropy web site is http: //www.astropy.org. We also acknowledge the use of STILTS and TOPCAT tools (Taylor 2005).

\section{References}

Abazajian, K. N., Adelman-McCarthy, J. K., Agüeros, M. A., et al. 2009, ApJS, 182,543

Alam, S., Albareti, F. D., Allende Prieto, C., et al. 2015, ApJS, 219, 12

Astropy Collaboration, Robitaille, T. P., Tollerud, E. J., et al. 2013, A\&A, 558, A33

Bacon, R., Copin, Y., Monnet, G., et al. 2001, MNRAS, 326, 23

Baldwin, J. A., Phillips, M. M., \& Terlevich, R. 1981, PASP, 93, 5

Blanton, M. R., Lupton, R. H., Schlegel, D. J., et al. 2005, ApJ, 631, 208

Brinchmann, J., Charlot, S., Heckman, T. M., et al. 2004a, ArXiv e-prints [arXiv: astro-ph/0406220]

Brinchmann, J., Charlot, S., White, S. D. M., et al. 2004b, MNRAS, 351, 1151

Buat, V., Boselli, A., Gavazzi, G., \& Bonfanti, C. 2002, A\&A, 383, 801

Bundy, K., Bershady, M. A., Law, D. R., et al. 2015, ApJ, 798, 7

Cardelli, J. A., Clayton, G. C., \& Mathis, J. S. 1989, ApJ, 345, 245

Casado, J., Ascasibar, Y., Gavilán, M., et al. 2015, MNRAS, 451, 888

Catalán-Torrecilla, C., Gil de Paz, A., Castillo-Morales, A., et al. 2015, A\&A, 584, A87

Chabrier, G. 2003, ApJ, 586, L133

Charlot, S., Kauffmann, G., Longhetti, M., et al. 2002, MNRAS, 330, 876

Cid Fernandes, R., Stasińska, G., Mateus, A., \& Vale Asari, N. 2011, MNRAS, 413, 1687

Colless, M., Dalton, G., Maddox, S., et al. 2001, MNRAS, 328, 1039

Contini, T., Garilli, B., Le Fèvre, O., et al. 2012, A\&A, 539, A91

Dahlem, M. 1997, PASP, 109, 1298

Drake, A. B., Simpson, C., Collins, C. A., et al. 2013, MNRAS, 433, 796

Drake, A. B., Simpson, C., Baldry, I. K., et al. 2015, MNRAS, 454, 2015

Driver, S. P., Hill, D. T., Kelvin, L. S., et al. 2011, MNRAS, 413, 971

Dutton, A. A., van den Bosch, F. C., \& Dekel, A. 2010, MNRAS, 405, 1690

Elbaz, D., Daddi, E., Le Borgne, D., et al. 2007, A\&A, 468, 33

García-Benito, R., Zibetti, S., Sánchez, S. F., et al. 2015, A\&A, 576, A135

Guidi, G., Scannapieco, C., Walcher, J., \& Gallazzi, A. 2016, MNRAS, 462, 2046

Hirashita, H., Buat, V., \& Inoue, A. K. 2003, A\&A, 410, 83

Hopkins, A. M., Miller, C. J., Nichol, R. C., et al. 2003, ApJ, 599, 971

Hopkins, A. M., Driver, S. P., Brough, S., et al. 2013, MNRAS, 430, 2047

Hunter, J. D. 2007, Comput. Sci. Eng., 9, 90

Husemann, B., Jahnke, K., Sánchez, S. F., et al. 2013, A\&A, 549, A87

Iglesias-Páramo, J., Buat, V., Takeuchi, T. T., et al. 2006, ApJS, 164, 38

Iglesias-Páramo, J., Vílchez, J. M., Galbany, L., et al. 2013, A\&A, 553, L7

Iglesias-Páramo, J., Vílchez, J. M., Rosales-Ortega, F. F., et al. 2016, ApJ, 826, 71

Ivezić, Ž., Connolly, A., Vanderplas, J., \& Gray, A. 2014, in Statistics, Data Mining and Machine Learning in Astronomy (Princeton University Press)

Jones, E., Oliphant, T., Peterson, P., et al. 2001, Scipy: Open source scientific tools for Python, http: //www . spicy . org [accessed 2016-03-22]

Kauffmann, G., Heckman, T. M., Tremonti, C., et al. 2003a, MNRAS, 346, 1055

Kauffmann, G., Heckman, T. M., White, S. D. M., et al. 2003b, MNRAS, 341, 33

Kehrig, C., Monreal-Ibero, A., Papaderos, P., et al. 2012, A\&A, 540, A11

Kehrig, C., Vílchez, J. M., Pérez-Montero, E., et al. 2016, MNRAS, 459, 2992

Kennicutt, Jr., R. C. 1998, ApJ, 498, 541

Kennicutt, R. C., \& Evans, N. J. 2012, ARA\&A, 50, 531

Kennicutt, Jr., R. C., Lee, J. C., Funes, J. G. S. J., Sakai, S., \& Akiyama, S. 2008 ApJS, 178, 247

Kennicutt, Jr., R. C., Hao, C.-N., Calzetti, D., et al. 2009, ApJ, 703, 1672

Kewley, L. J., \& Ellison, S. L. 2008, ApJ, 681, 1183

Kewley, L. J., Dopita, M. A., Sutherland, R. S., Heisler, C. A., \& Trevena, J. 2001, ApJ, 556, 121

Kewley, L. J., Jansen, R. A., \& Geller, M. J. 2005, PASP, 117, 227

Koyama, Y., Kodama, T., Hayashi, M., et al. 2015, MNRAS, 453, 879

Kroupa, P. 2001, MNRAS, 322, 231

Le Fèvre, O., Vettolani, G., Garilli, B., et al. 2005, A\&A, 439, 845

Leslie, S. K., Kewley, L. J., Sanders, D. B., \& Lee, N. 2016, MNRAS, 455, L82 
Madau, P., Ferguson, H. C., Dickinson, M. E., et al. 1996, MNRAS, 283, 1388

Mannucci, F., Cresci, G., Maiolino, R., Marconi, A., \& Gnerucci, A. 2010, MNRAS, 408, 2115

Marchesini, D., van Dokkum, P. G., Förster Schreiber, N. M., et al. 2009, ApJ, 701,1765

Mast, D., Rosales-Ortega, F. F., Sánchez, S. F., et al. 2014, A\&A, 561, A129

McKinney, W. 2010, in Proc. 9th Python in Science Conference, eds. S. van der

Walt, \& J. Millman, 51

Momcheva, I. G., Lee, J. C., Ly, C., et al. 2013, AJ, 145, 47

Nakamura, O., Fukugita, M., Brinkmann, J., \& Schneider, D. P. 2004, AJ, 127, 2511

Nelson, D., Pillepich, A., Genel, S., et al. 2015, Astron. Comp., 13, 12

Noeske, K. G., Weiner, B. J., Faber, S. M., et al. 2007, ApJ, 660, L43

O’Donnell, J. E. 1994, ApJ, 422, 158

Osterbrock, D. E. 1989, Astrophysics of Gaseous Nebulae and Active Galactic Nuclei (USA: University Science Books)

Peng, Y.-J., Lilly, S. J., Kovač, K., et al. 2010, ApJ, 721, 193

Pérez, F., \& Granger, B. E. 2007, Comp. Sci. Eng., 9, 21

Pilyugin, L. S., Lara-López, M. A., Grebel, E. K., et al. 2013, MNRAS, 432, 1217

Relaño, M., Lisenfeld, U., Vilchez, J. M., \& Battaner, E. 2006, A\&A, 452, 413

Renzini, A., \& Peng, Y.-J. 2015, ApJ, 801, L29

Richards, S. N., Bryant, J. J., Croom, S. M., et al. 2016, MNRAS, 455, 2826

Rodighiero, G., Daddi, E., Baronchelli, I., et al. 2011, ApJ, 739, L40

Rosales-Ortega, F. F., Kennicutt, R. C., Sánchez, S. F., et al. 2010, MNRAS, 405, 735

Salim, S., Rich, R. M., Charlot, S., et al. 2007, ApJS, 173, 267
Salpeter, E. E. 1955, ApJ, 121, 161

Sánchez, S. F., Kennicutt, R. C., Gil de Paz, A., et al. 2012, A\&A, 538, A8

Schlegel, D. J., Finkbeiner, D. P., \& Davis, M. 1998, ApJ, 500, 525

Sparre, M., Hayward, C. C., Springel, V., et al. 2015, MNRAS, 447, 3548

Speagle, J. S., Steinhardt, C. L., Capak, P. L., \& Silverman, J. D. 2014, ApJS, 214,15

Storey, P. J., \& Hummer, D. G. 1995, MNRAS, 272, 41

Strauss, M. A., Weinberg, D. H., Lupton, R. H., et al. 2002, AJ, 124, 1810

Taylor, M. B. 2005, in Astronomical Data Analysis Software and Systems XIV, eds. P. Shopbell, M. Britton, \& R. Ebert, ASP Conf. Ser., 347, 29

Tissera, P. B., Pedrosa, S. E., Sillero, E., \& Vilchez, J. M. 2016, MNRAS, 456, 2982

Tremonti, C. A., Heckman, T. M., Kauffmann, G., et al. 2004, ApJ, 613, 898

Trujillo, I., Aguerri, J. A. L., Cepa, J., \& Gutiérrez, C. M. 2001, MNRAS, 321, 269

Van Der Walt, S., Colbert, S. C., \& Varoquaux, G. 2011, Comput. Sci. Eng., 13, 22

van der Wel, A., Chang, Y.-Y., Bell, E. F., et al. 2014, ApJ, 792, L6

Vanderplas, J., Connolly, A., Ivezić, Ž., \& Gray, A. 2012, in Conf. on Intelligent Data Understanding (CIDU), 47

Veilleux, S., \& Osterbrock, D. E. 1987, ApJS, 63, 295

Walcher, C. J., Wisotzki, L., Bekeraité, S., et al. 2014, A\&A, 569, A1

Whitaker, K. E., van Dokkum, P. G., Brammer, G., \& Franx, M. 2012, ApJ, 754, L29

York, D. G., Adelman, J., Anderson, Jr., J. E., et al. 2000, AJ, 120, 1579

Zahid, H. J., Dima, G. I., Kewley, L. J., Erb, D. K., \& Davé, R. 2012, ApJ, 757, 54 


\section{Appendix A: The SFR- $M_{\star}$ and galaxy size- $M_{\star}$ relations of SDSS and CALIFA star-forming galaxies}

In order to strengthen the relevance between CALIFA and SDSS galaxies, we have compared CALIFA star-forming galaxies in the $S F R-M_{\star}$ and size- $M_{\star}$ diagrams with the SDSS galaxies (Figs. A.1 and A.2, respectively). Figure A.1 shows the $S F R-M_{\star}$ for SDSS and CALIFA star-forming galaxies. The SFRs values for the CALIFA galaxies were computed consistently following the methodology presented in Sect. 3.2 of this work, using the data for the star-forming galaxies in Catalán-Torrecilla et al. (priv. comm.). The linear fit to the running median of the aperture-corrected $S F R-M_{\star}$ distribution for the complete sample $(0.005 \leq z \leq 0.22)$, and for the first redshift range considered $(0.005 \leq z<0.05)$ are shown, together with the median values of SFR and $M_{\star}$ along five stellar mass bins for CALIFA star-forming galaxies; equitable number of galaxies have been considered in each bin.

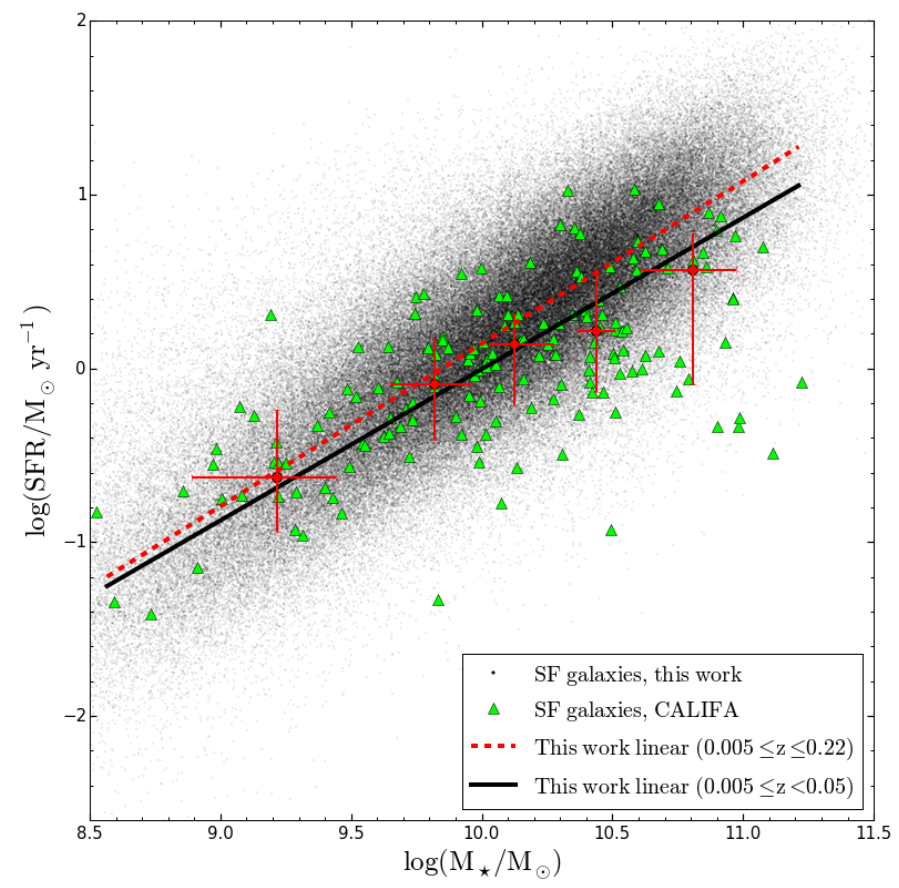

Fig. A.1. $S F R-M_{\star}$ relation for SDSS (this work) and CALIFA (Catalán-Torrecilla et al. 2015) star-forming galaxies. $S F R-M_{\star}$ fit to the running median for bins of 2000 objects obtained in this work for the complete sample (red dashed line) and for bins of 1000 objects in the range of $z$ between $0.005 \leq z<0.05$ (black solid line). Red dots represent the median values of SFR and $M_{\star}$ for CALIFA star-forming galaxies along five stellar mass bins for an equitable number of galaxies in each bin. The error bars in $x$ - and $y$-axis represent the $\pm 1 \sigma$ confidence interval for stellar mass and SFR, respectively.

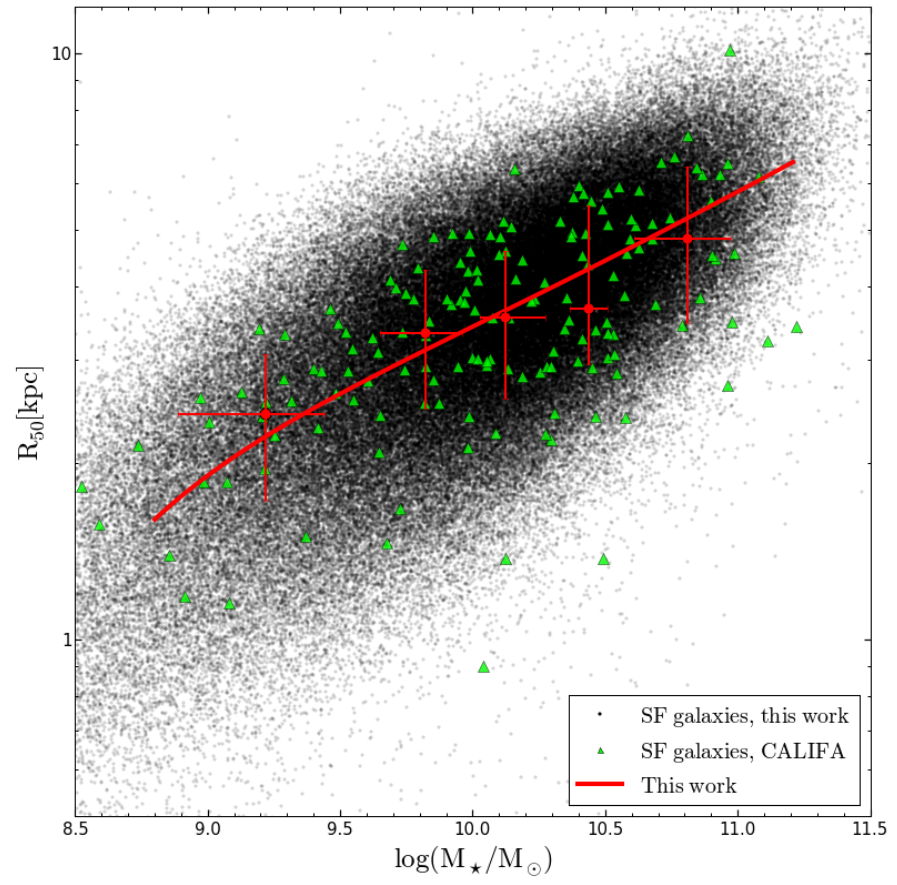

Fig. A.2. Size- $M_{\star}$ relation for SDSS (this work) and CALIFA (Catalán-Torrecilla et al. 2015) star-forming galaxies. Red solid line represents the linear fit to the running median for bins of 2000 objects in the SDSS star-forming galaxies. Red dots represent the median values of size and $M_{\star}$ for CALIFA star-forming galaxies along five stellar mass bins for an equitable number of galaxies in each bin. The error bars in $x$ - and $y$-axis represent the $\pm 1 \sigma$ confidence interval for stellar mass and size, respectively.

Figure A.2 shows the size- $M_{\star}$ for SDSS and CALIFA starforming galaxies. The linear fit to the running median of the size$M_{\star}$ distribution for the complete sample $(0.005 \leq z \leq 0.22)$ and the median values of size and $M_{\star}$ along five stellar mass bins for CALIFA star-forming galaxies are shown; equitable number of galaxies have been considered in each bin.

From the figures we can see that the median values of the SFR of CALIFA galaxies are representatives with the results obtained in this work for (nearby $0.005 \leq z<0.05$ ) SDSS galaxies over the whole range of galaxy mass. Likewise, the median values of the galaxy size of CALIFA galaxies are visibly consistent with the SDSS galaxies distribution. Taking into account these results, we can consider CALIFA star-forming galaxies as representative of the SDSS star-forming galaxies sample used in this work. 